TEXT and rectangles in blue will NOT show on printed copy

Type final title of thesis or dissertation (M.S. and Ph.D.) below. If your title has changed since your submitted an Application for Graduate Degree, notify Graduate Office.

Microfluidic Devices For Characterizing the Viability of Cryptosporidia Oocysts by Dielectrophoresis

A Thesis

Presented to

the faculty of the School of Engineering and Applied Science

University of Virginia

in partial fulfillment

of the requirements for the degree

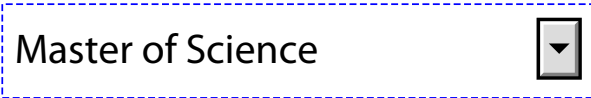

by

Name

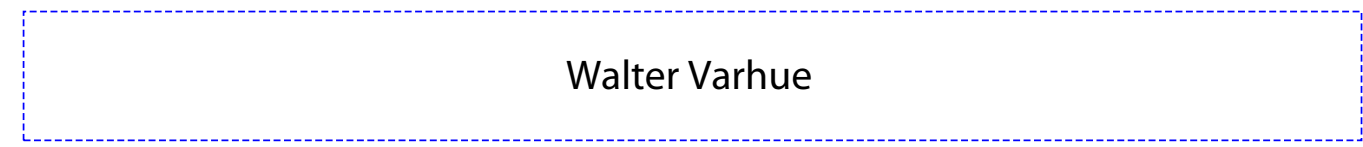

Month degree is awarded

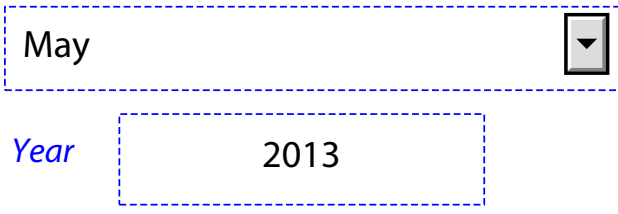


APPROVAL SHEET

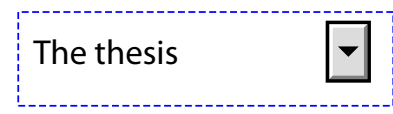

is submitted in partial fulfillment of the requirements

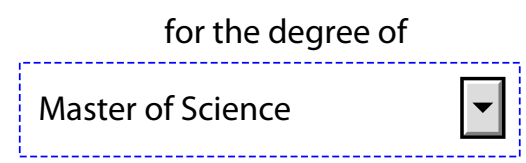

AUTHOR signature

The thesis has been read and approved by the examining committee:

Please insert committee member names below:

Nathan Swami

Advisor

John Bean

David Green

Accepted for the School of Engineering and Applied Science:

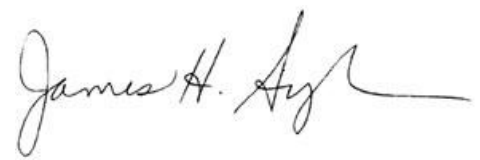

Dean, School of Engineering and Applied Science

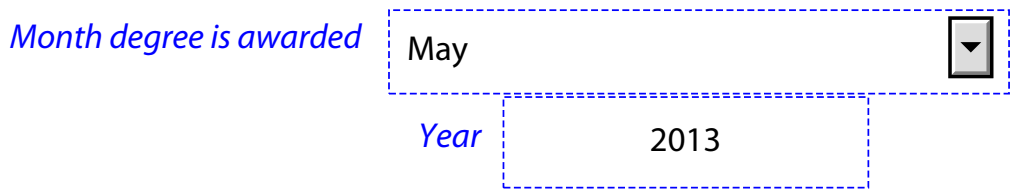




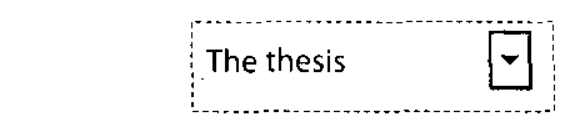

is submitted in partial fulfillment of the requirements

for the degree of

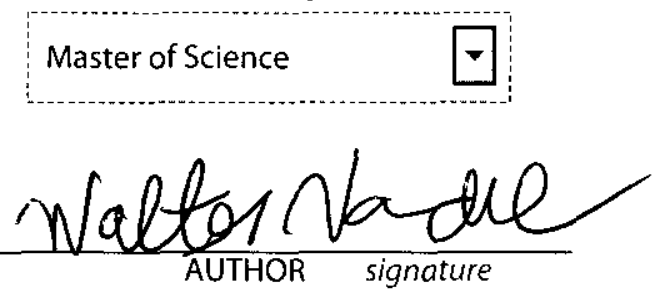

The thesis has been read and approved by the examining committee: Please insert committee member names below:

\section{Nathan Swami}

Advisor

John Bean

David Green

Accepted for the School of Engineering and Applied Science:

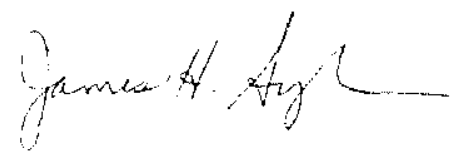

Dean, School of Engineering and Applied Science

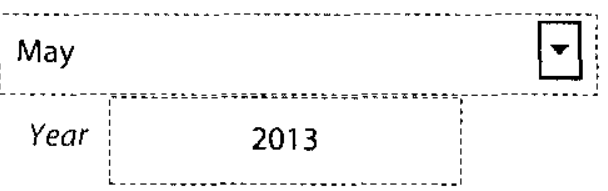




\title{
Microfluidic devices for characterizing the viability of Cryptosporidia oocysts by dielectrophoresis
}

\section{Walter Varhue}

\begin{abstract}
:
Cryptosporidium parvum is a dangerous parasitic organism that attacks the small intestine of infected individuals. This water born pathogen causes the severe intestinal disease cryptosporidiosis, which has no direct treatment and can often prove fatal to immunocompromised individuals. While its viability is modified by heat treatment, Cryptosporidia oocysts have proven to be significantly resistant to chlorine-based disinfectants. It is necessary to research more effective disinfecting agents that can be easily and safely dispersed under these circumstances.
\end{abstract}

The search for such an agent would be aided by the availability of a fast, inexpensive, and reliable method for characterizing the viability of Cryptosporidia oocysts. Towards this, we investigate microfluidic device designs utilizing the electrokinetic phenomena of dielectrophoresis. Dielectrophoresis, or DEP, is on the translation of a polarized particle under non-uniform AC electric field. The direction of this force is caused by differences in the Maxwell-Wagner polarization of the particle and its surrounding fluidic medium as described by the frequency dependent Clausius Mossotti factor. By analyzing differences in the direction of the DEP force at specific AC field frequencies, difference between two particles can be discerned. In the case of Cryptosporidia oocysts, we hypothesize that these discernable differences include modifications to the viability of the parasites, including changes to their oocyst wall and cytoplasm by disinfecting agents.

In this paper we will optimize microfluidic device designs for dielectrophoretic characterization of the modifications to viability of the oocysts after disinfection treatment. Through this, several design considerations for microfluidic devices utilizing this phenomenon will be highlighted. We will also analyze the design, fabrication, and experimental operation of three microfluidic devices that utilize DEP as a detection method for Cryptosporidium viability. Three device designs, including a set of quadra-pole electrodes, the diamond post constriction device and the single channel constriction device were utilized. 


\section{Cryptosporidium}

Cryptosporidium parvum is a water-borne pathogen infecting mammals. This infection can be fatal within immune-compromised individuals. Upon ingestion, the spore, or oocyst, stage of Cryptosporidium's life cycle will target its host's small intestine releasing infective sporozites. This causes the acute intestinal infection called cryptosporidiosis, whose primary symptoms include severe diarrhea and abdominal pain. There is currently no effective therapy that can directly treat Cryptosporidiosis, with all known treatments focusing exclusively on the infections symptoms.[2] In a healthy immune-competent adult, this infection proves self-limiting and usually resolves itself within one to two weeks.[3] In an immune-compromised individual this infection usually proves far more severe, leading to a high mortality rate in such persons.[2]

Due to the lack of a direct treatment options for Cryptosporidium infection and its high mortality rate among infected immune-compromised individuals, there must be an increased emphasis on the prevention of infection. Prevention, in this case, can best be achieved through the eradication of the infectious Cryptosordium occysts from water used by potential hosts. Drinking water carrying the pathogen can be made potable relatively easily through either heat treatment[7] or filtration. Larger volumes of water used for swimming or bathing (a common vector for cryptosporidiosis) can prove more problematic as the thick walled cryptosporidium oocysts are largely resistant to the common chlorine based disinfectants currently used for such purposes.[3] It is necessary to develop an alternative methods of disinfection, which can be widely and safely deployed in large volumes of water.

Research into such alternative methods, however, is somewhat hampered by the lack of a fast, inexpensive, and reliable method for testing the viability and infectivity of the Cryptosprodia Oocysts. Currently the primary method used for this purpose is the mouse 
infectivity study.[3] This method calls for the observation of laboratory animals introduced to a potentially infectious sample over a period of time. By quantifying the amount of Oocysts shed by the study animals it is possible to judge the infectivity of the water sample. While accurate, this mouse model is both time consuming and expensive, calling for weeks of preparation and observation. Furthermore, a concentration of at least $10^{7}$ oocysts $/ \mathrm{mL}$ is required to obtain quantitative data on infectivity, whereas as few as 10 oocysts can cause substantial infections within mammals.[2] Other faster methods of testing, such as propidium iodide (PI) florescent stains, have further drawbacks. While PI has been shown to accurately detect damage of the oocyst wall, by methods such as heat treatment, it is unable to detect damages to the spores in the cytoplasm. More sensitive molecular methods of detection, based on analysis of the nucleic acids (DNA and RNA) are destructive, time consuming and require trained technicians. For this purpose a new method of viability testing must be developed which has improved sensitivity over the current mouse infectivity study, and can be applied in a routine manner for real-time monitoring of water samples following disinfection treatments. One platform proposed for this purpose is the electrokinetic method of Dielectrophoresis or DEP.

\section{Dielectrophoresis:}

Dielectrophoresis (DEP) is an electrokinetic method which causes the translation of a polarized particle in an acquiesce medium under a non-uniform electric field. Electrokinetic manipulation has long been used for the characterization of biological particles through the common experimental method of gel track electrophoresis. This common laboratory technique utilizes a uniform DC field to separate particles of different electrophoretic mobilities (electrostatic versus viscous drag force) in a gel medium. Unlike electrophoresis, which causes 
translation due to the induced like charges under a DC field, DEP uses the polarization of a particle and its surrounding medium to make electrokinetic distinctions.

In order to understand these methods of electrokinetic manipulation, one must first examine the electrical properties of a particle in a fluidic medium. Biological particles contain bound charge carrying ions.[6] These charges may exist both inside the particle and on its surface. Often there will be an imbalance in the charges bound on the surface, resulting net surface charge. When submerged in aqueous solution these bound charges attract opposite free charges from the solution. These free charges make up the double layer, i.e. a shell of counter

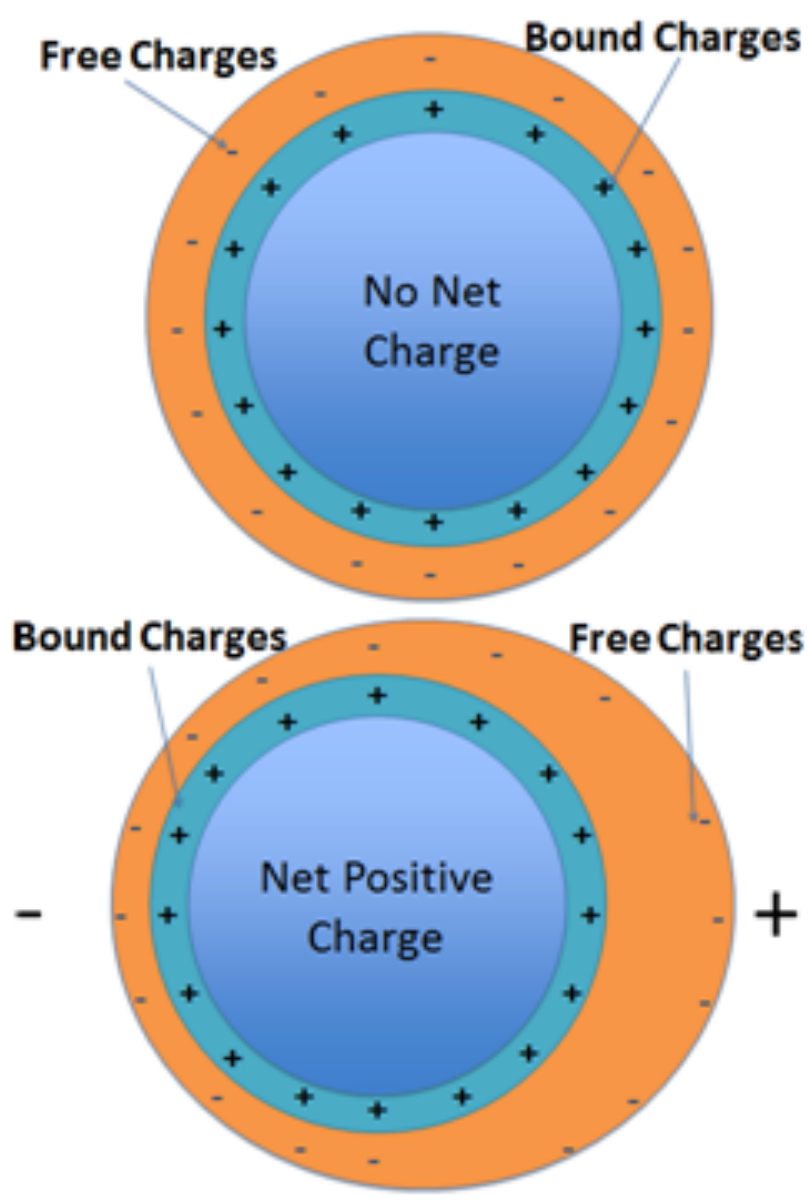

Figure 1: A: (Top) counter charges from the solution balancing charges surrounding the are attracted to the bound surface charges of a particle forming a double layer. There is no resulting net charge on the particle. B: (Bottom) an applied DC particle resulting in a net charge of zero field pulls the free charges away from one side of the particle resulting in double layer warping. There is a is demonstrated in figure 1a. When a resulting net positive charge on the particle[6]

in a

an field of sufficient strength is introduced, the free charges that make up the outer portion of the double layer will be pulled away causing the outer portion of the double layer to warp.[6] The bound charges on the surface are unable to be manipulated in such a way, leaving a net charge on the particle. As a result of this net charge, the DC field exerts a force on the particle. Depending 
upon the size of the particle, the viscosity of the surrounding medium, and the particles surface charges, this force can be used to separate and categorize different biological particles. However in many cases the differences between size and charge characteristics of different particles is minimal, requiring a long particle travel distances to determine any distinction. For use as a method to detect changes in a particle, such as viability in a cell, electrophoresis is a poor choice, as the size of the particle will remain consistent. In these cases, the faster and more selective phenomena of DEP may be applicable as it does not rely on the charge of the particle, but on its ability to polarize in an electric field at characteristic frequencies.

To understand DEP we must look at what occurs when a particle, similar to the one described above, is affected by a non-uniform AC electric field. When introduced to a low frequency $\mathrm{AC}$ electric field the direction of double layer warping will oscillate with the direction of the electric field resulting in a diffusional movement of counter-ions with the switching dipoles on the particle. . This warping however is not instantaneous. As the frequency is increased the extent of the particle's movement due to electrophoresis decreases until a threshold frequency is reached where the double layer can no longer react fast enough to the changing electric field.[6] This leaves the particle with a neutral net charge and prevents motion due to electrophoresis. There are other electrical phenomena that can react faster than the double layers warping, including interfacial or Maxwell Wagner Polarization.[6] When an electric field is exerted on a particle, the charges in the particle will attempt to align counter to the electric field resulting in the formation of a dipole. Similarly, the medium around the particle will attempt to screen the charges due to the dipole, resulting in opposite charges forming at the interface of the particle and the medium. In the case where the particle is 'more polarizable' than the medium $\left(\varepsilon_{\mathrm{p}}{ }^{*}>\varepsilon_{\mathrm{m}}{ }^{*}\right)$ the dipole formed inside the particle will be stronger resulting in a net dipole moment 
that is parallel to the electric field. In the case where the medium is 'more polarizable' the charges at the surface of the particle will be greater than those inside the particle, resulting in a net dipole moment that is counter to the electric field. In a uniform electric field the coulomb forces exerted on both ends of the dipole are equal and there is no net force exerted on the particle.[1] In a non-uniform electric field, however, the coulomb force on one end of the dipole will be greater, resulting in a net force either towards or away from a point of high field, depending on polarizability of particle $\left(\varepsilon_{\mathrm{p}}{ }^{*}\right)$ versus the medium $\left(\varepsilon_{\mathrm{m}}{ }^{*}\right)$. In the case where the dipole moment is parallel to the electric field, the charge of the pole closest to the high field point will be opposite of the high field point. This will result in a net force on the particle that is directed towards the high field point. This case is classified as positive DEP and is characterized by the movement of the particle towards a high field point.[1] In the case where the dipole moment is antiparallel to the

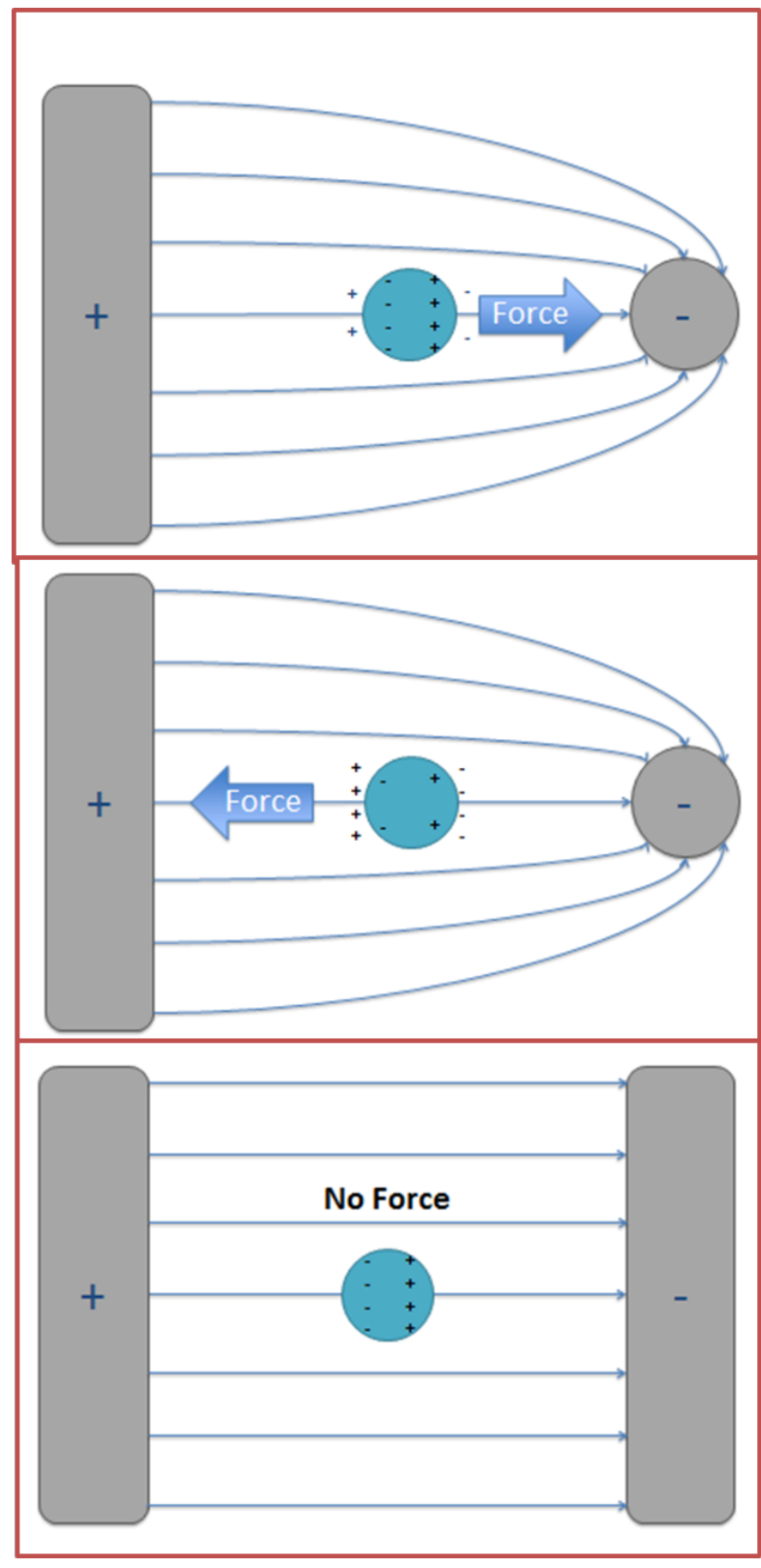

Figure 2: A: (top) A polarized particle under a non-uniform field where $\left(\varepsilon_{\mathrm{p}}{ }^{*}>\varepsilon_{\mathrm{m}}{ }^{*}\right)$ experiences a force directed towards the high field point, i.e. positive DEP. $\mathrm{B}($ middle $)$ ) A polarized particle under a non-uniform field where $\left(\varepsilon_{\mathrm{p}}{ }^{*}<\varepsilon_{\mathrm{m}}{ }^{*}\right)$ experiences a force directed away from the high field point, i.e. negative DEP. C:(bottom) A polarized particle under a uniform field, no force is applied from DEP[1]

field, the pole closest to the high field 
point will carry the same charge as the high field point, resulting in a force directed away from it. This case is classified as negative DEP and is characterized by the repulsion of the particle away from a high field point.[1]

The net DEP force is given by: $\mathrm{F}=\mathrm{p} . \nabla \mathrm{E}$. For a spherical particle in a non-uniform electric field (E), the time averaged DEP force is given by.

$$
F_{\mathrm{DEP}}=2 \pi \varepsilon_{\mathrm{m}} R^{3} C M(E \nabla \mathrm{E})
$$

equation $1[8]$

Where $\mathrm{R}$ is the radius of the particle, $\mathrm{E}$ is the amplitude of the electric field, $\nabla \mathrm{E}$ expresses the gradient of the electric field, $\varepsilon_{\mathrm{m}}$ is the absolute permittivity of the medium, and CM is the Clausius-Mossotti factor. The Clausius-Mossotti factor is a component which compares the polarizablity of the particle to that of the medium and can be given by

$$
C M=\left(\frac{\varepsilon_{p}^{*}(\omega)-\varepsilon_{m}^{*}(\omega)}{\varepsilon_{p}^{*}(\omega)+2 \varepsilon_{m}^{*}(\omega)}\right)
$$

equation 2[1]

where $\varepsilon^{*}(\omega)$ is the frequency dependent complex permittivity given by

$$
\varepsilon^{*}(\omega)=\varepsilon-j \frac{\sigma}{\omega}
$$

equation 3[1]

Where $\varepsilon$ is the permittivity of the particle or medium, $\sigma$ is it's conductivity, $\mathrm{j}$ is the square root of -1 , and $\omega$ is the frequency of the applied AC electric field. The sign of the Clausius -Mossotti 
factor will be the determining component of the direction of coulomb force applied on the particle by DEP. Equation two shows that a positive Clausius-Mossotti factor will occur when the complex permittivity of the particle is higher (i.e the particle is more polarizable) than that of the medium. This case will result in positive DEP. When the reverse is true, the ClausiusMossotti factor will be negative, leading to negative DEP.

As is suggested by equation 3 , when a low frequency field is applied the effective permittivity's value will be largely determined by the conductivity $(\sigma)[9]$. As the frequency is increased, conductivity will play less of a determining role in the value of the effective permittivity until, eventually, the value will mainly be determined by the material's permittivity (ع).[9] Since alterations to the functionality of a cells are likely to change its dielectric properties, the resulting change in the Clausius-Mossotti factor will alter the movement of the cell under a non-uniform field. Hence by monitoring how the magnitude and direction of the DEP force on a polarized bio-particle responds to frequency changes, alterations to it can be quantified in realtime.

Since changes in the frequency of the electric field influence the Clausius-Mossotti factor it is possible for the same particle in the same medium to exhibit both positive and negative DEP at different frequencies. Often this change will occur when the effective permittivity changes from being primarily determined by the conductance of the material to that determined by permittivity of the material. The frequency at which the $\mathrm{CM}$ factor changes from positive to negative (or vice versa) for a specific particle in a specific medium is called its crossover frequency.[10] When a medium with a consistent conductivity and permittivity is used changes in this crossover frequency will suggest modifications affecting the dielectric properties such as the conductivity, porosity, and deformability of the tested particles. Moreover if two particles 
with different crossover frequencies are placed in the same medium, then a non-uniform AC field with a frequency in between their crossover frequencies may be used to separate them.[10] Utilizing this method of distinction, differences in biological particles can quickly and easily be detected by analyzing their DEP frequency responses.

When investigating this method of distinction for use in viability testing of Cryptosporidia oocysts, several factors need to be taken into account. Paramount among these is the dielectric nature of both infectious and non-infectious Oocysts. Unlike many of the particles previously discussed, microorganisms, such as cryptosporidium oocysts, are not made of a single material with a uniform dielectric constant or conductivity. Instead they are composite structures made of different materials with differing degrees of polarizability at each material interface.[8] To simplify these cases, it is often useful to apply a shell model.[11] This method depicts the various components of a cell as regions with different conductive and dielectric properties, often taking the form

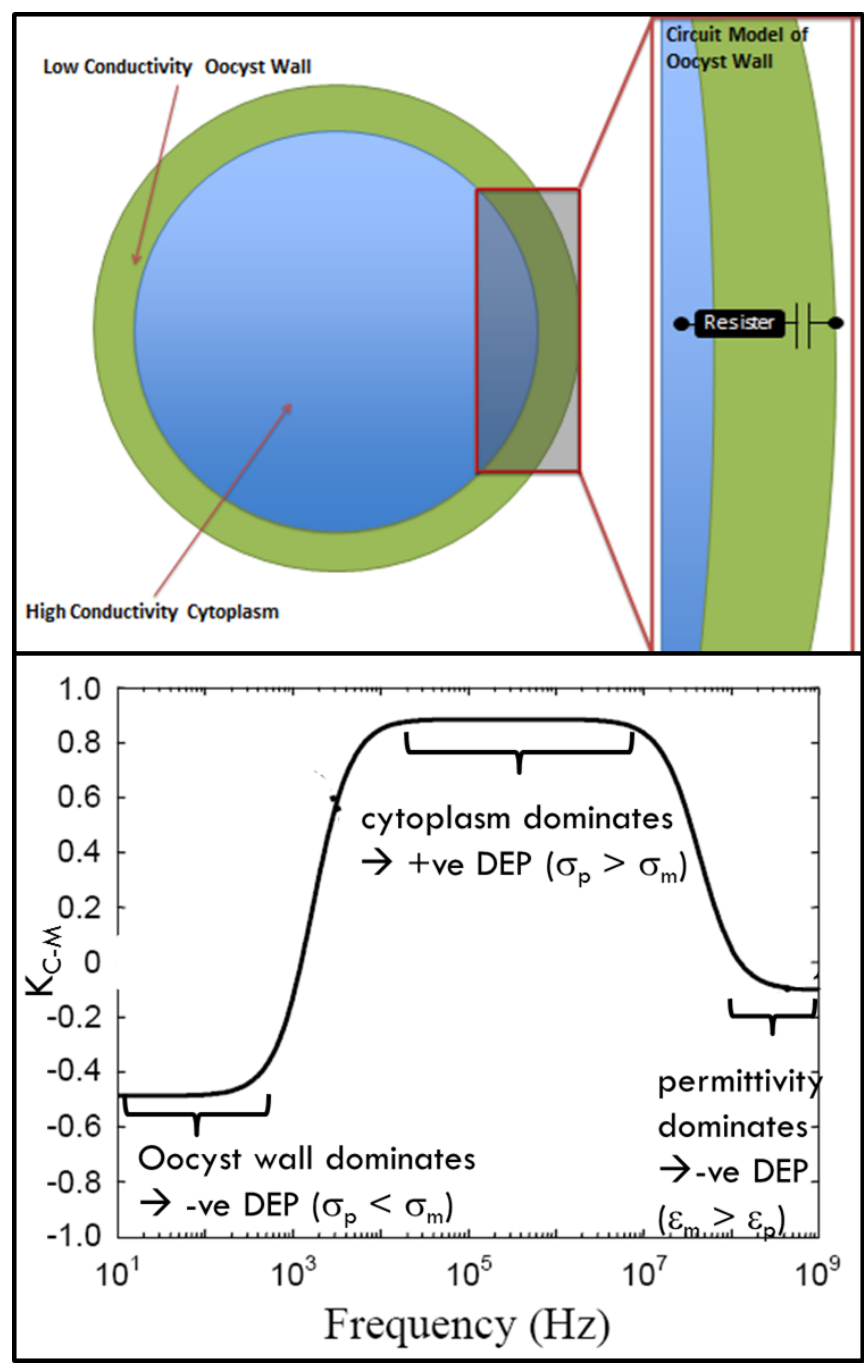

Figure 3: A (top) Shell Model of Cryptosporidium including RC circuit model of the oocyst wall B: (Bottom) Clausious-Mossoti facto versus frequency. Adapted from AC Electrokinetics: colloids and nanoparticles by Morgan and Green[4]

of concentric spheres, ellipses or cylinders. For this purpose a viable Cryptosporidium Oocyst 
can be described as a relatively simple cell having two primary components. These are the sporozite supporting cytoplasm surrounded by a thick protective oocyst wall. To estimate the dielectric nature of this structure a low conductivity spherical shell (oocyst wall) surrounding a higher conductivity core (cytoplasm) can be used.[8] This configuration will have an interesting effect on the frequency response of the CM factor. At low frequencies the capacitive nature of the cell wall will shield the applied field from the high conductivity cytoplasm at the interior, thereby preventing it from affecting the $\mathrm{CM}$ factor. In this case the low conductivity of the oocyst wall with respect to the surrounding medium $\left(\sigma_{\text {wall }}<\sigma_{m}\right)$ will cause the oocyst to exhibit negative DEP. As the frequency is raised, however, this capacitive shielding will diminish to eventually allow the higher conductivity cytoplasm to affect the net DEP force. The high conductivity of the cytoplasm with respect to the medium $\left(\sigma_{\text {cytoplasm }}>\sigma_{\mathrm{m}}\right)$ will cause positive DEP at a critical frequency, reflecting the first crossover frequency. Eventually at even higher frequencies, the CM factor will change from being primarily determined by the material's conductivity to that determined by permittivity. Since the aqueous medium has a higher permittivity than the cytoplasm, the oocysts will undergo negative DEP, resulting in a second cross over frequency. This model will exhibit changes upon modifications to the viability of the oocysts. Heat treatment, the most common form of disinfection, will likely damage the oocyst wall. The higher RC time constant due to the increase in impedance of the capacitor due to the oocyst wall causes a shift of the first DEP crossover to lower frequencies. [8, 12] In some other cases where the oocyst wall has been damaged but is still partially intact, the conductivity is not substantially altered, thereby causing no substantial DEP $\left(\sigma_{\text {wall }} \sim \sigma_{\mathrm{m}}\right)$ or even negative DEP over a wider frequency range $\left(\sigma_{\text {wall }}<\sigma_{\mathrm{m}}\right)$. In cases where the oocyst wall has been more effectively destroyed, no significant capacitive shielding is possible. This results in positive DEP at low 
frequencies and no DEP at higher frequencies due to loss of cytoplasm conductivity. It has been suggested that other methods of disinfection may not damage the cell wall. Instead they may attack the sporozited in the cytoplasm, thereby changing its dielectric properties.[13] In these cases the oocyst will still undergo negative DEP at low frequencies, where the oocyst wall acts as a capacitor. At higher frequencies, the force and direction of the DEP will shift due to the change in the cytoplasm. Due to these different changes in frequency response it is suggested that DEP may be used to isolate the portion of the cell affected by a particular disinfection agent.

\section{Device Design:}

When looking to design devices for use in cell viability testing for disinfection analysis through dielectrophoretic response, several design considerations need to be taken into account. To limit the necessary sample volume, enhance the applied electric field, enable highly-parallel measurements, and to improve portability it is beneficial to fabricate such devices on a microfluidic platform. These devices will have two primary functions: (1) the ability to effectively generate a non-uniform electric field in an aqueous medium; and (2) to allow a clear distinction between the positive and negative DEP responses of a sample. The first function will be important as it will greatly affect the magnitude, but not the direction, of the DEP force applied to the tested particle. Equation 1 demonstrates that this effect on the force will not only be governed by the magnitude of the electric field but also by its gradient. Therefore it is important to find ways to improve both the field magnitude at its high field point and the field gradient around this point.

The second function will allow spatial distinction between positive and negative DEP behavior. One method to achieve this is through a system that utilizes DEP trapping. Trapping is where the device manipulates the electric field in such a way as to create well-defined regions of 
high and low field. These trapping regions will confine particles undergoing either positive or negative DEP to spatially separated regions of the microfluidic device. This method of spatial segregation will allow the user of such a device to make a clear distinction between groups of particles undergoing either positive or negative DEP. Furthermore, the magnitude of the DEP force may be ascertained by measuring the velocity of the particles towards or away from the high field region, for positive or negative DEP, respectively. As an optical system, such as an inverted microscope and camera, will be used to make record observations, it is also important that these trapping regions are visible. This will entail using transparent materials in these regions to allow light and the image to be accurately transferred.

The use of cryptosporidium samples requires several further considerations. The diameter of cryptosporidium oocysts ranges between $4.5 \mathrm{um}$ to $5.5 \mathrm{um}[2]$. This size will govern the minimum dimensions of the microfluidic devices. Furthermore, the size of these particles will necessitate the leveling and isolation of any experimental setup to prevent movement due to gravity or environmental vibration. Also the electrical properties of cryptosporidium are different from several other cells with which this technology has already been proven. Specifically cryptosporidiums cytoplasm has been estimated as $0.052 \mathrm{~S} / \mathrm{m}[14]$, while e.coli has been estimated at .1 S/m[14]. This suggests that higher electric field magnitudes or gradients will be needed to generate the same DEP trapping force for Cryptosporidia oocysts versus E. coli cells. 


\section{Quadrapole Device:}

The most common method of inducing an inhomogeneous electric field in a fluidic medium is through the use of micro-gap electrodes. Figure 4 demonstrate this approach using a configuration of two dissimilar electrodes. In this case the gap between the electrodes is used to generate the field gradient necessary for DEP. As these figures suggest the field lines in this configuration will constrict around the smaller electrode. As this will be the region with the highest concentration of field lines it will be the high-field point where a particle undergoing DEP will either be drawn (positive DEP) to or repulsed (negative DEP) from. In this way the direction of the force can be ascertained.

However, while this design does localize polarized particles for positive DEP trapping at the edge of the smaller electrode, the localization region for particles under negative DEP is not well defined.

This flaw can be corrected by utilizing four electrodes in a quadrapole design. In this case, four similar micro-patterned electrodes are arranged in a bilaterally symmetric pattern as shown in figure 5.[1] When placed in a microfluidic chamber filled with the sample particles and medium, these electrodes will be energized by several external leads such that

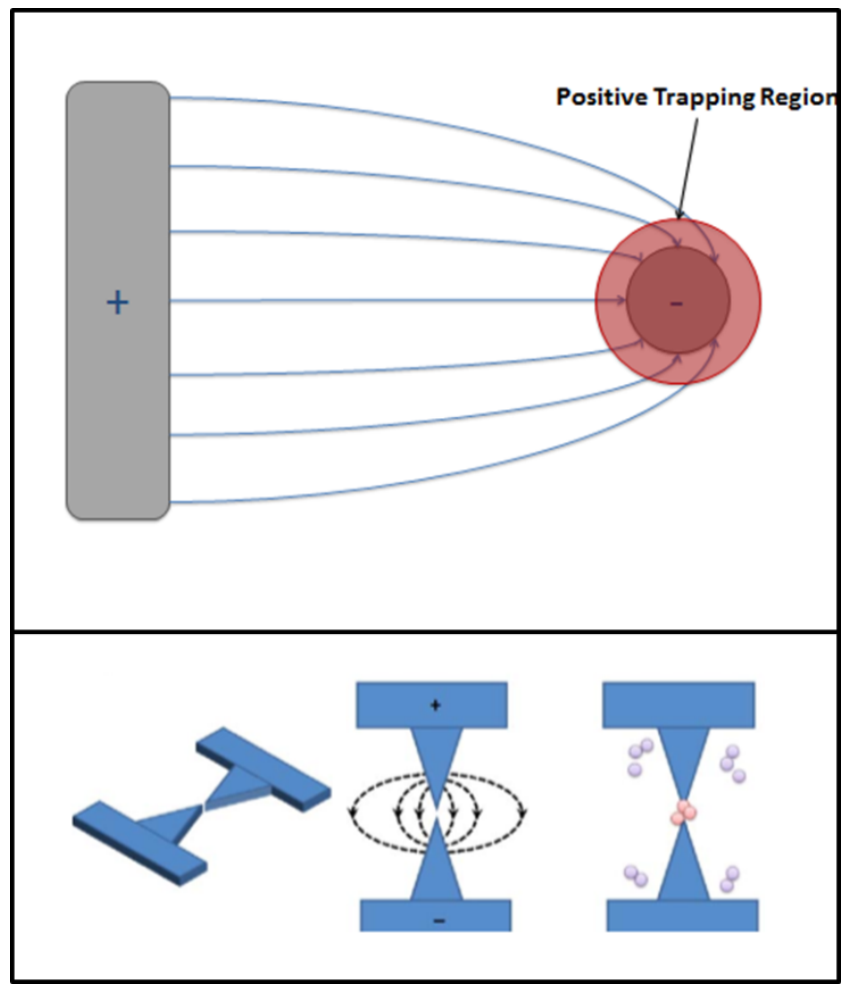

Figure 4 A: (Top) A two electrode design utilizing dissimilar electrodes to induce a non-uniform field B:(bottom) A two electrode design from C. Zhang's "Dielectrophoresis for manipulation of micro/nano particles in microfluidic systems “ [1]

they each face an electrode with the same polarity. The resulting field lines are depicted in figure 
5A. Instead of relying on dissimilar electrode dimensions to generate a field gradient these electrodes will utilize a sharp tip on each electrode to create a high field point as is shown in figure 5B. A low field region will also be created at the center of the shown device. This design integrates both, an easy way to generate field gradients, and to discern between positive or negative trapping.

\section{Experimental Methods: Quadrapole}

Our quardrapole device utilized four sharp tipped micro-patterned electrodes spaced 80 microns apart. In the trapping regions the patterned electrode will be tapered to allow for better visibility in the area of interest, before converging to a sharp point. Beyond the trapping regions the width of the gold electrode pattern expanded to mitigate the effects of scoring in the patterned gold that may occur from handling during fabrication and experimental setup. To fabricate this design, a common gold liftoff method was used to pattern

the microelectrodes on a glass substrate as is shown if figure 6 . Photolithography was used to

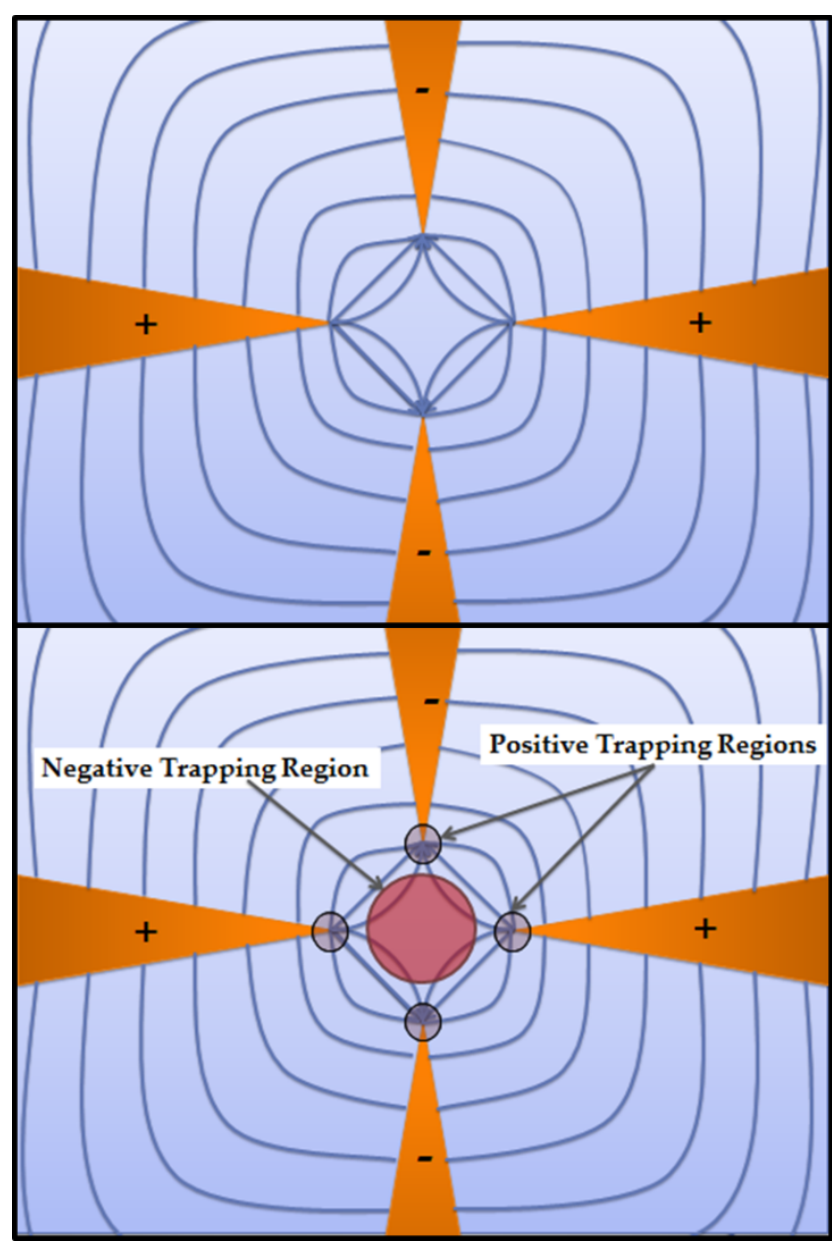

Figure 5: A: (top) The field lines generated by the quadrapole electrode. B: Negative and Positive trapping regions in a quadrapole design. form an inverse of the pattern on a glass sample slide with AZ 5209 positive photo resist. Electron beam vapor deposition was then used to deposit a conducting layer composed of a $\mathrm{Ti}$ adhesion layer $(\sim 20 \mathrm{~nm})$ and $80 \mathrm{~nm}$ gold layer. The excess gold and titanium was then removed 
using an acetone lift-off bath with sonication. Electrical connections were then soldered to the contact pads to allow for easy experimental setup.

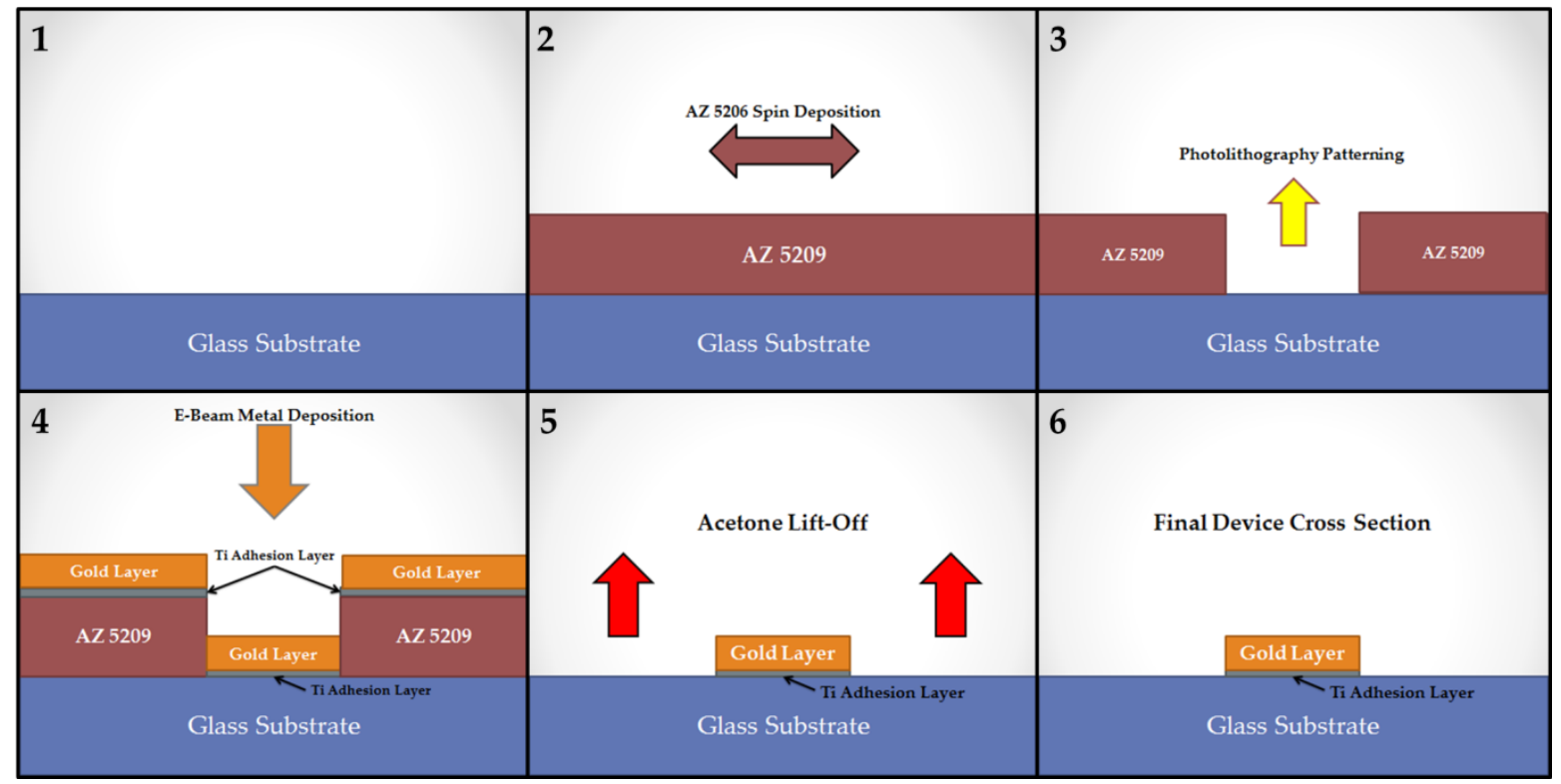

Figure 6: Process flow for quadrapole device fabrication

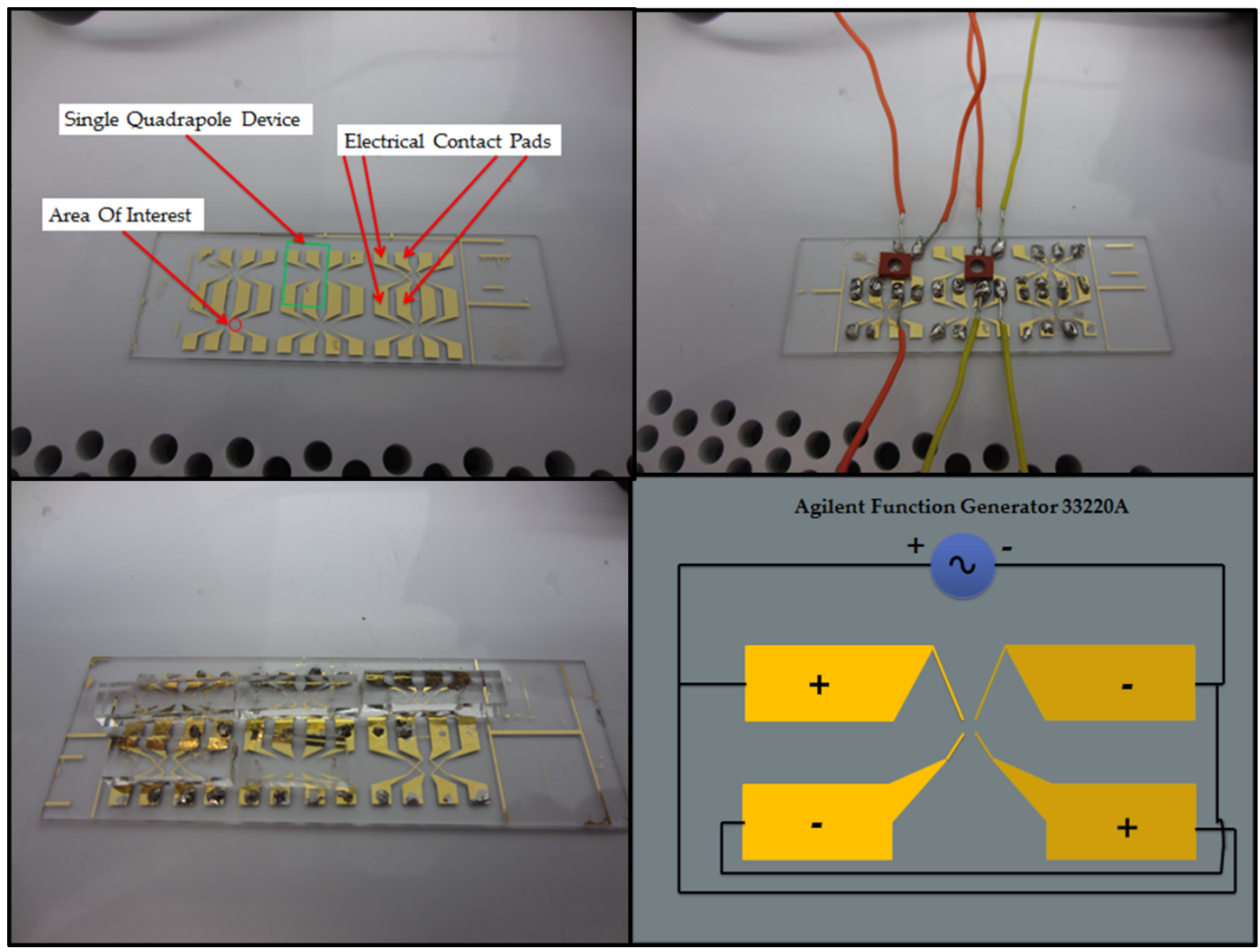

Figure 7: A: (top left) micropatterned Quadrapole electrodes B: (top right) Quadraploe device with silicone gaskets C: (bottom left) Quadrapole device with bonded PDMS micro-chambers D: (bottom right) Electrical connections for device 
To introduce the sample particles and medium, a microfluidic chamber was constructed on a micromolded polymer layer of PDMS (poly di-methyl siloxane) and then aligned and bonded over the quadrapole tips. Two methods have been used to fabricate the fluidic chamber

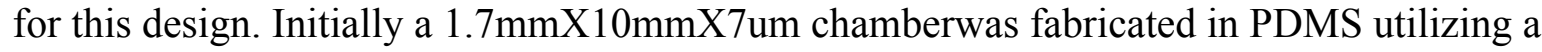
micro molding process. This chamber had inlet and outlet holes drilled through the PDMS and was aligned and bonded to the glass slide through oxygen plasma bonding. However this system proved to be vulnerable to leaks along the gold electrical connections due to the inability of PDMS inability to strongly bond to gold. Furthermore, collapses in the chamber could result due to its high aspect ratio. A simpler method was based on a silicone gaskets with a double sided adhesive that was applied to the area around the quadrapole tips to form a well for the fluid. The well could be filled with the sample and then a small cover glass slide was placed on top of the well to prevent evaporation. To apply the electric field a signal generator was connected to the soldered leads as shown in figure $7 \mathrm{~d}$. Data was collected using a Zeiss inverted microscope system with a Hamamatsu EMCCD camera.

\section{Results: Quadrapole}

We begin with experiments on DEP characterization of untreated Cryptosporidia oocysts in DI water $\left(\sigma_{\mathrm{m}} \sim 0.1-0.3 \mathrm{mS} / \mathrm{m}\right)$. Positive DEP trapping at frequencies below $100 \mathrm{kHz}$ cannot be easily discerned using quadrapole devices due to the disruption from electrolysis and electrohydrodynamics on particles trapped by positive DEP. Hence, the earliest frequency where DEP behavior was discernible was at $200 \mathrm{kHz}$, where a weak level of negative DEP trapping was apparent, as per Figure 4. Beginning from frequencies above $350 \mathrm{kHz}$, weak positive DEP trapping was apparent and this grows to strong positive trapping in the frequency range from $400 \mathrm{kHz}$ to $10 \mathrm{MHz}$. This suggests the first crossover frequency (negative to positive DEP) 
occurred between $200 \mathrm{khz}$ and $350 \mathrm{khz}$ for untreated viable Cryptosporidia oocysts. For oocytes disinfected through a low temperature $\left(80^{\circ} \mathrm{C}\right)$ heat treatment process, the DEP behavior using quadrapole devices demonstrated weak negative DEP trapping from $10 \mathrm{kHz}$ to $100 \mathrm{kHz}$ and a stronger level of negative DEP trapping in the $200 \mathrm{kHz}$ to $1 \mathrm{MHz}$ frequency range.

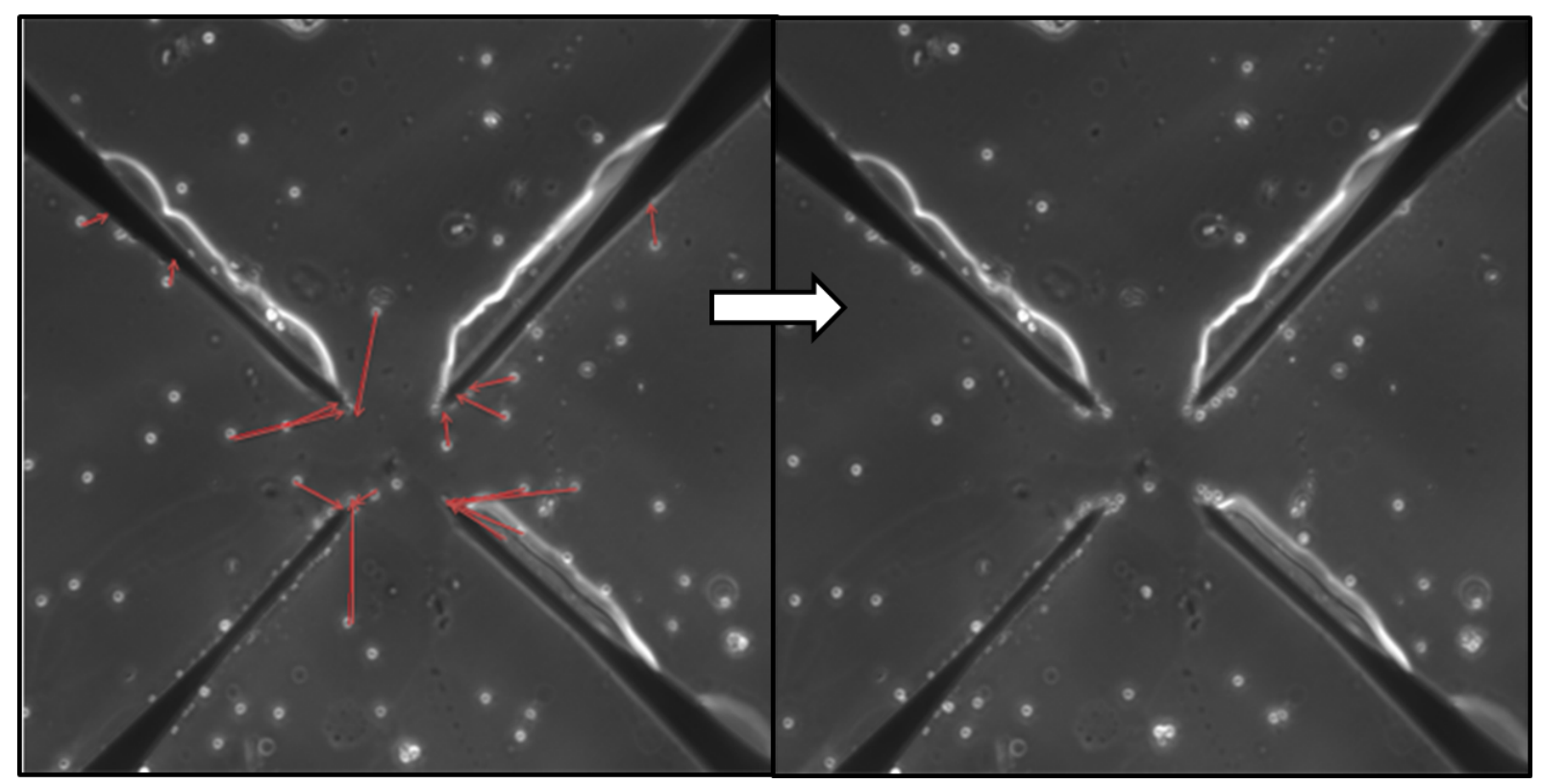

Figure 8: Quadrapole Device exhibiting strong positive DEP. The red lines indicate the direction of translation of the cryptosporidium prior to being trapped.

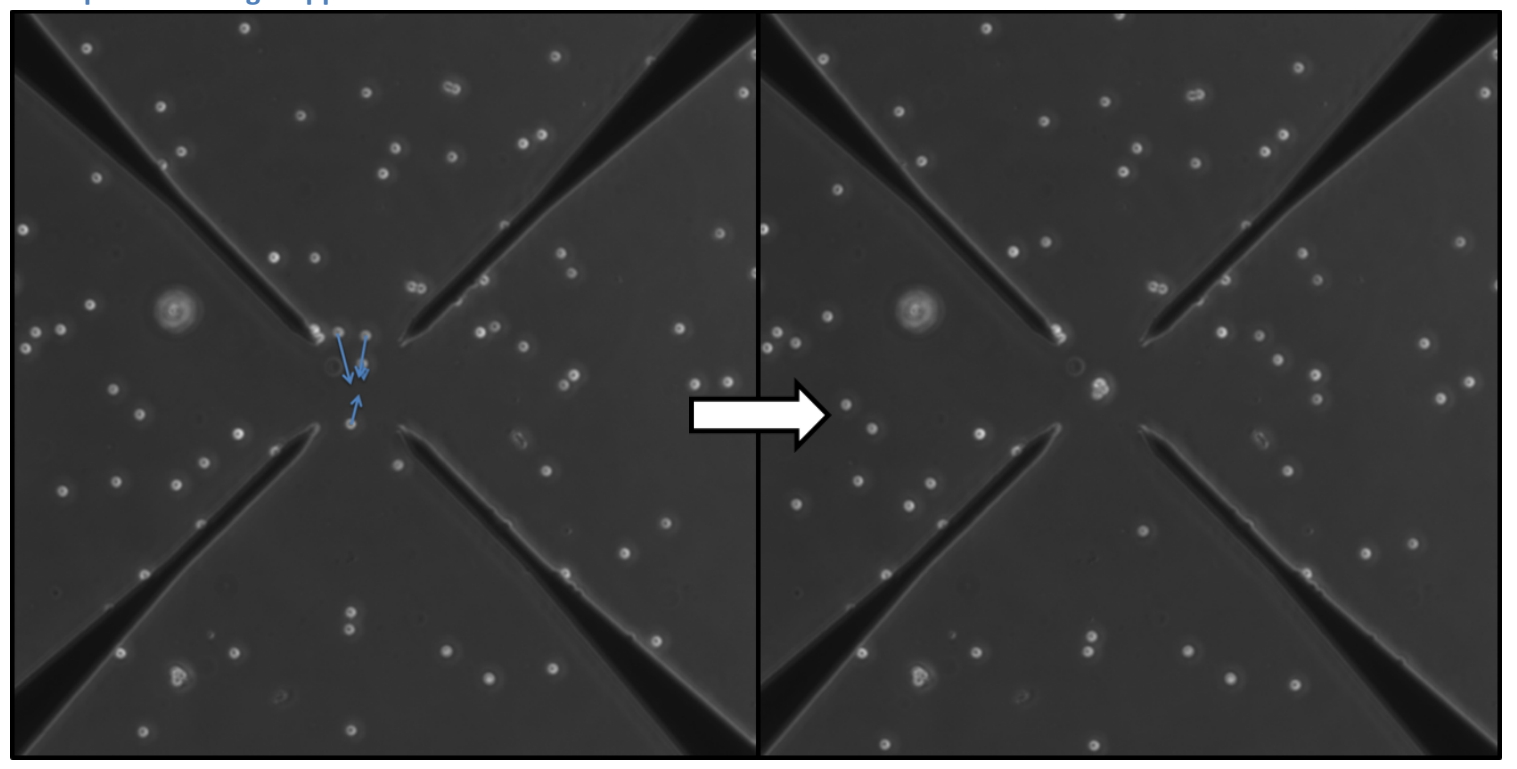

Figure 9: Quadraploe Device exhibiting strong Negative DEP. The blue lines indicate the direction of translation of the cryptosporidium prior to being trapped. 


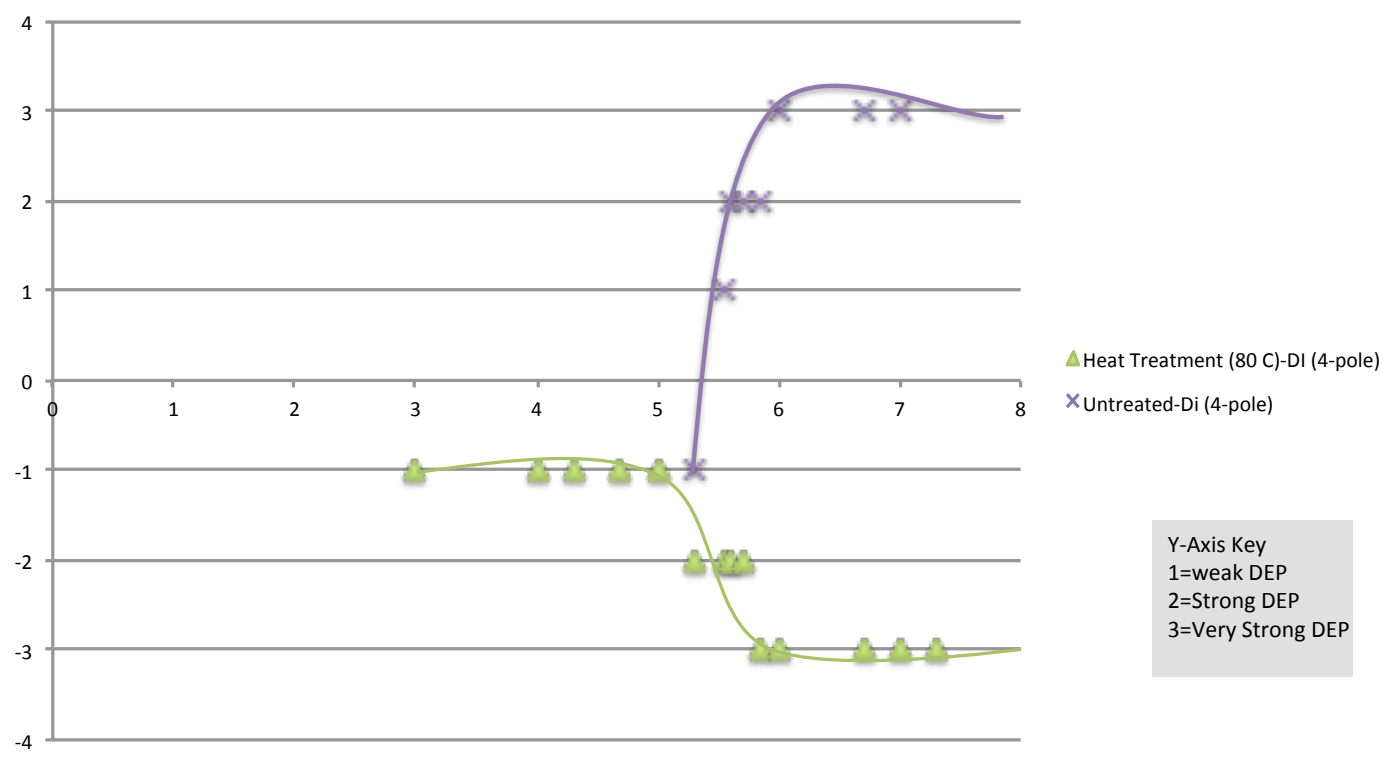

Figure 10: qualitative DEP strength and direction version the log scale of the applied AC electric field for untreated and Heat treated cryptosporidium oocytes in a quadrapole device.

While DEP trapping using quadrapole devices was successfully achieved with live and heat-treated Cryptosporidia oocysts, several limitations of the design were also discovered. At low frequencies the close proximity of the electrodes led to electrolysis of the medium. This would facilitate the formation of bubbles separating the electrodes from the medium essentially acting as an electrical open circuit. This largely prevented any discernable data from be collected at lower frequencies. Also the obscuring nature of the gold on titanium electrodes proved disruptive to the experimental process. These opaque electrodes may have prevented accurate data collection during positive DEP trapping as they obscured a portion of the trapping area. In some experiments, the positive DEP trapping regions were not localized at the high-field points at the sharp tips of the electrodes, but also along their entire electrode edge. This likely occurred due to the sharp angle left over from the lift off process step during microfabrication.

Furthermore, positive DEP trapping of the oocyst at the high-field point can cause damage to the cell due to the current flow from its direct contact with the electrode[6], which can skew results with viability cell tests. 
The greatest weakness of the quadrapole design, however, is the relatively low spatial extent of the region where trapping may occur and the inability of the field to draw new cells towards this trapping area. While effective at trapping the cells in the square area of interest defined by the tips of the quadrapole electrodes, this high-field tends to affect a relatively low number of cells. Increasing the distance between the microelectrodes could expand the area of interest, but this would result in a substantially lower field density, thereby lowering the DEP trapping force. Since the field is relatively uniform beyond this high-field region at the electrode tips, there is no discernable DEP behavior elsewhere within the device. Furthermore, there is no method to constantly draw new cells towards this region. This, combined with the possible damage done to viable cells, limits the usefulness of the device for viability testing. This limited use is aggravated by the formation of bubbles and the adsorption of positively trapped cells to the high-field points of the electrodes, both of which act as insulators and prevent further trapping in those areas.

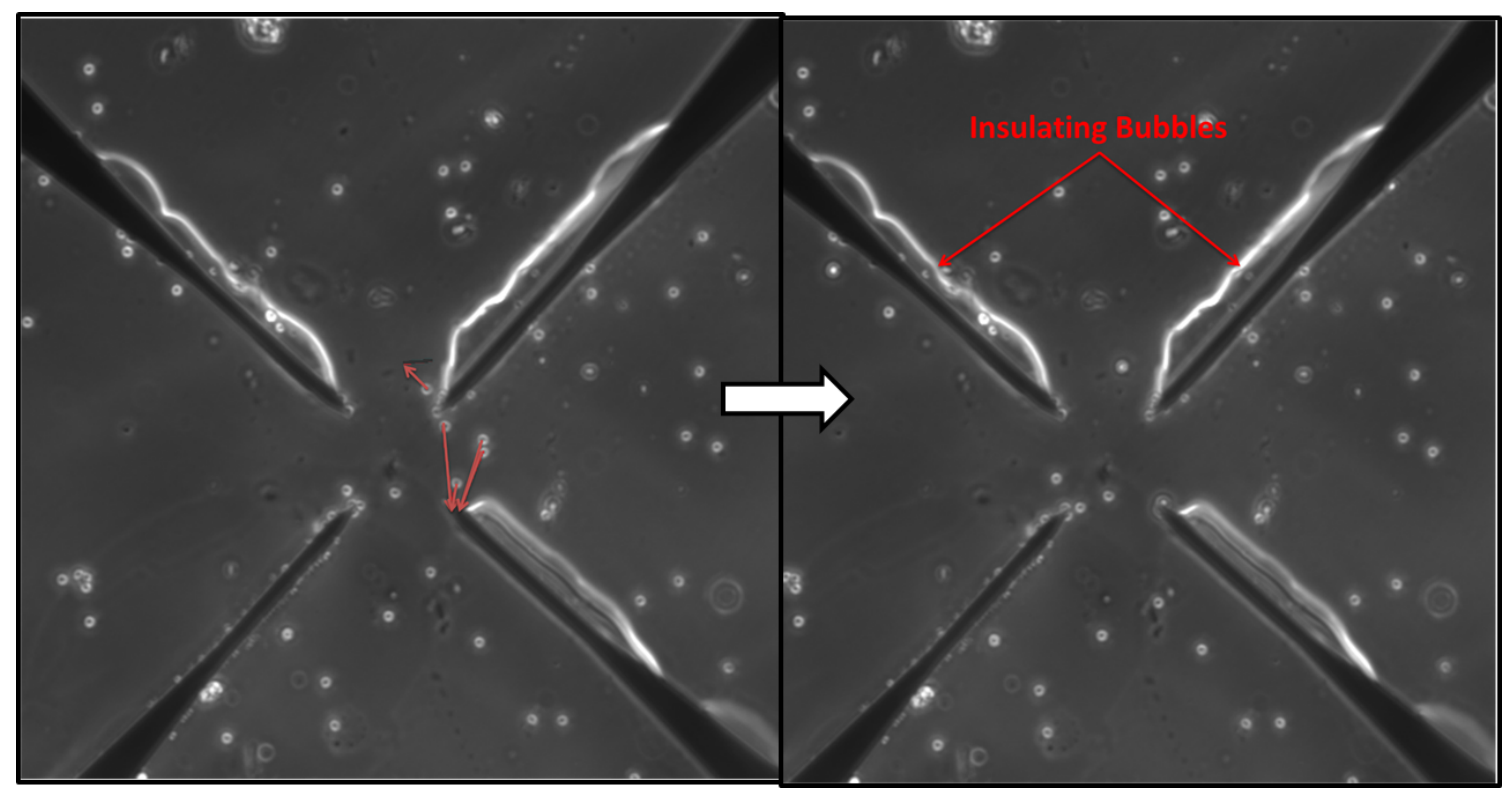

Figure 11: This figure depicts cells being unable to translate to their closest electrode due to adsorption of insulating cells and bubbles. Instead they are drawn a longer distance to a farther electrode. 


\section{Electrode-less DEP using insulating constrictions}

Some of the drawbacks of electrode based DEP devices can be overcome by an electrode-less DEP (EDEP) device utilizing insulating constriction tips to enhance the field. In this method the energizing electrodes are positioned at the device inlet and outlet, far from each other, with the trapping regions located at the tips of insulating constrictions within a microfluidic channel. Here the term electrode-less is used, since there is no conducting material in the trapping regions.[5] Instead, insulating structures, placed between the electrodes, localize the field within specific areas of a microfluidic device to create the high field points. Due to the increased distance between the electrodes, a much higher applied electric field is required. One such category of insulating structures that can be used for the manipulation of the electric field are triangular constrictions of an insulating material.[5] This method of electric field manipulation can be understood by exploring the equation for electric current density, which is given by

$$
J=\sigma E
$$

equation $4[5]$

Where $J$ is the current density, $\sigma$ is conductivity of the electrolyte medium, and $E$ is the electric field. If the constriction material is assumed to be perfectly insulating, then the current carrying ions from the electrolyte solution will be unable to penetrate the constriction walls. This will cause the current

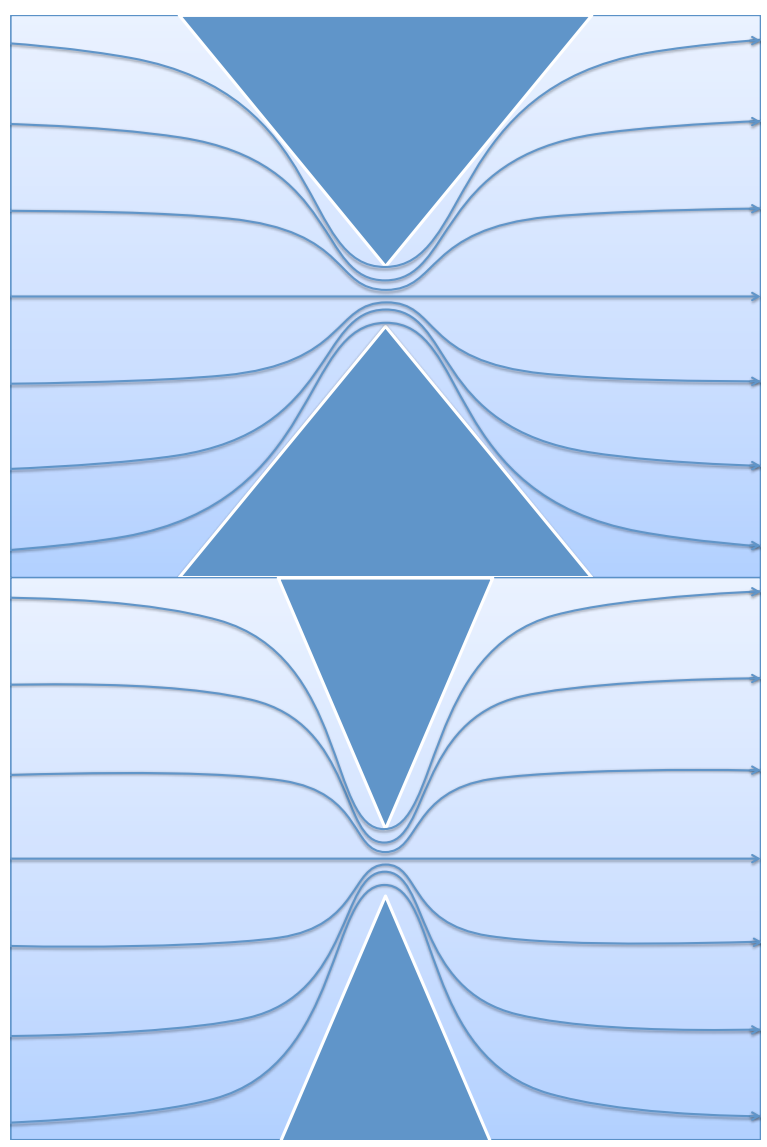

Figure 12: field lines in a constriction device[5] density to increase along the constricted areas, since 
the ions are forced through a narrower path.[5] This increase in current density causes an increase in the electric field at these points. This can also be demonstrated by electric field lines, which will be screened away from insulator in a parallel direction. As such they will bend and concentrate at the constriction tip of the insulating material, as is shown in Figure 12.

The dimensions of the constriction will be a determining factor of the field gradient and will also influence the magnitude at the high field point. In this device design, the high field point will be located at the tips of the constriction, as this will be the region with the highest concentration of electric field. The magnitude of this high field point will be determined primarily by the magnitude of the applied electric field and the gap size of the constrictions. The smaller the gap size is, the more concentrated the field will become in this region. The gradient of the applied field is determined by the sharpness or slope of the constriction. In this way, a sharper constriction will generate a steeper field gradient, since the change in the electric field will occur over as shorter length of channel.[5] While this will result in a higher DEP force it will also limit the extent of the non-uniform field, thereby reducing the spatial extent of the influence of DEP force.

Similar to the two electrode DEP method, this constriction design does not include any region of low field where negative DEP trapping can occur. However, negative DEP trapping can still

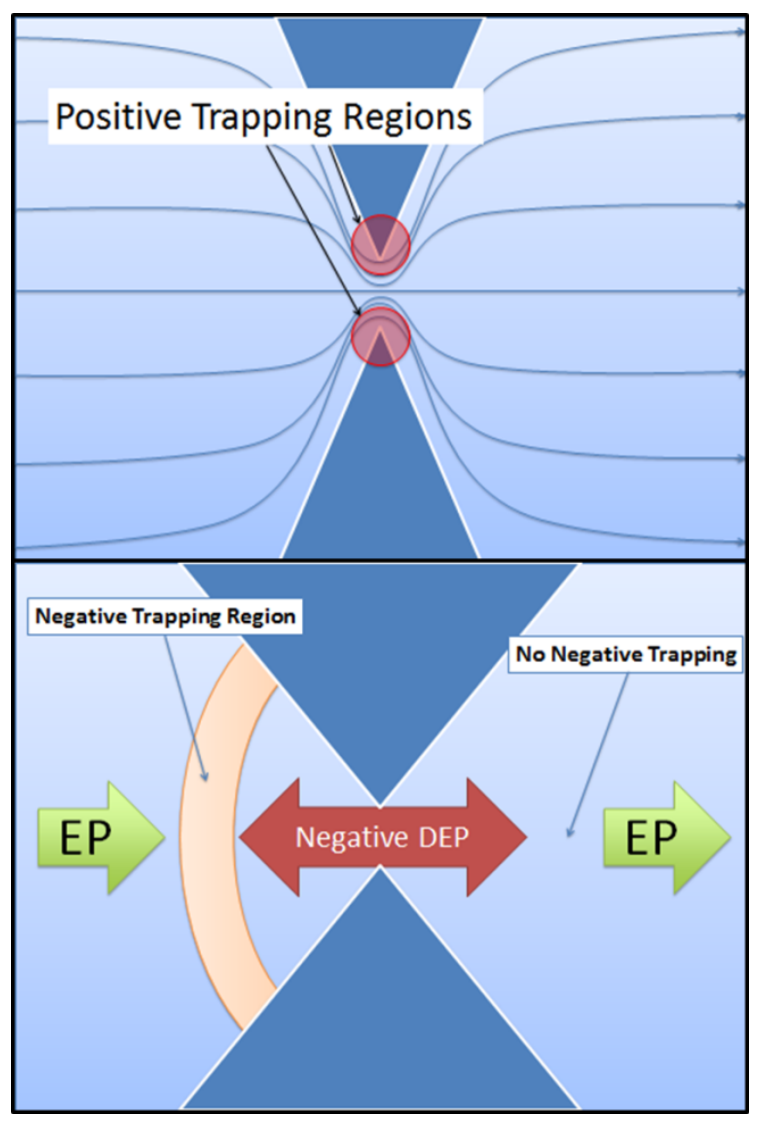

Figure 13:A:(top) positive trapping regions in a constriction Device. B:(bottom) Negative trapping region utilizing DC electrophoresis. Note the trapping region only occurs on the left side of the constriction. 
be made possible by introducing a minor offset DC voltage to the applied AC signal. This DC offset will cause the sample particles to experience electrophoretic force in one direction of the channel. This electrophoretic force will push the particle experiencing negative DEP towards the constriction until it is met by an equal but opposite negative DEP force. This creates a thin trapping region where these forces are equal. As the negative DEP force and the Electrophoretic force will only have opposite directons on one side of the constriction, trapping in this manner will be limited to that particular side of the constriction. Because electrophoresis is not limited to regions where the field is non-uniform, this method will allow for easy transport of particles outside of area affected by DEP to the constriction area. While not necessary for positive trapping, a DC offset can similarly be used to bring new target particles to the trapping region. This electrophoretic enhanced DEP trapping is one of the major highlights of this design as it allows for greater throughput.

\section{Design: Diamond Post Constriction E DEP Device}

Our first EDEP device for DEP

characterization of the viability of Cryptosporidia oocysts after disinfection treatments utilized an array of insulting diamond post structures in a microfluidic chamber. These posts were aligned to form several rows and columns along the breadth and width of the chamber to fabricate the insulating constrictions in proximity to one another. The spacing between posts

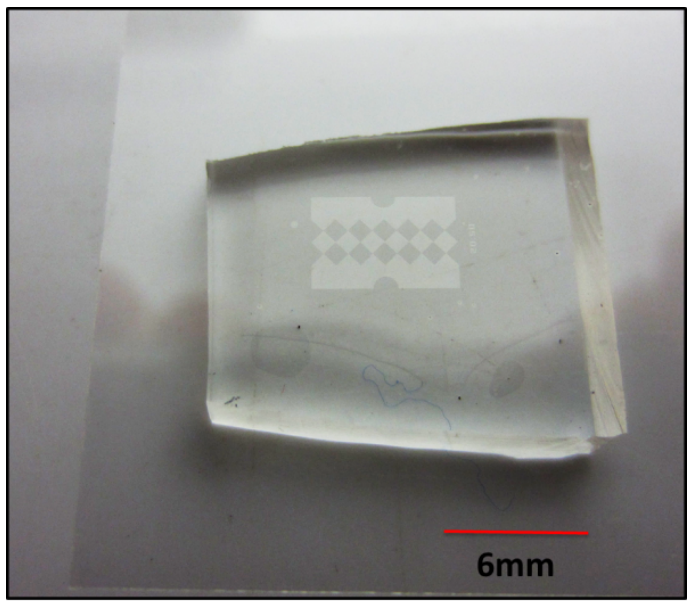

Figure 14: 7X2 diamond post design. Image contrast raised to $55 \%$ to increase visibility.

would define the minimum constriction width, while the size of the posts would define the 
maximum constriction width. By utilizing multiple parallel channels formed by these posts, this design would allow for high sample throughput and flexibility during device operation. To accommodate the large size of the Cryptosporidia oocysts (4.5um-5.5um), The constriction gaps were designed to be larger than $10 \mu \mathrm{m}$ to prevent clogging. Similarly the depth of the chamber was designed to be $8 \mu \mathrm{m}$. To simplify fabrication the inlet and outlet used to fill the microfluidic chamber would also act as the insertion point for the platinum electrodes used to introduce the electric field.

\section{Experimental Methods: Constriction Devices}

The diamond post devices were fabricated in PDMS using a micro moldingprocess with a SU8-5 silicon master.

Photolithography was used to pattern the negative photo resist SU8-5 on a 4 inch silicon wafer to form a raised 8 um high inverse structure of the desired microfluidic design. This patterned wafer served as the master for the molding process to fabricate the PDMS chips. During the molding process, un-cured 10:3 PDMS epoxy was poured into the

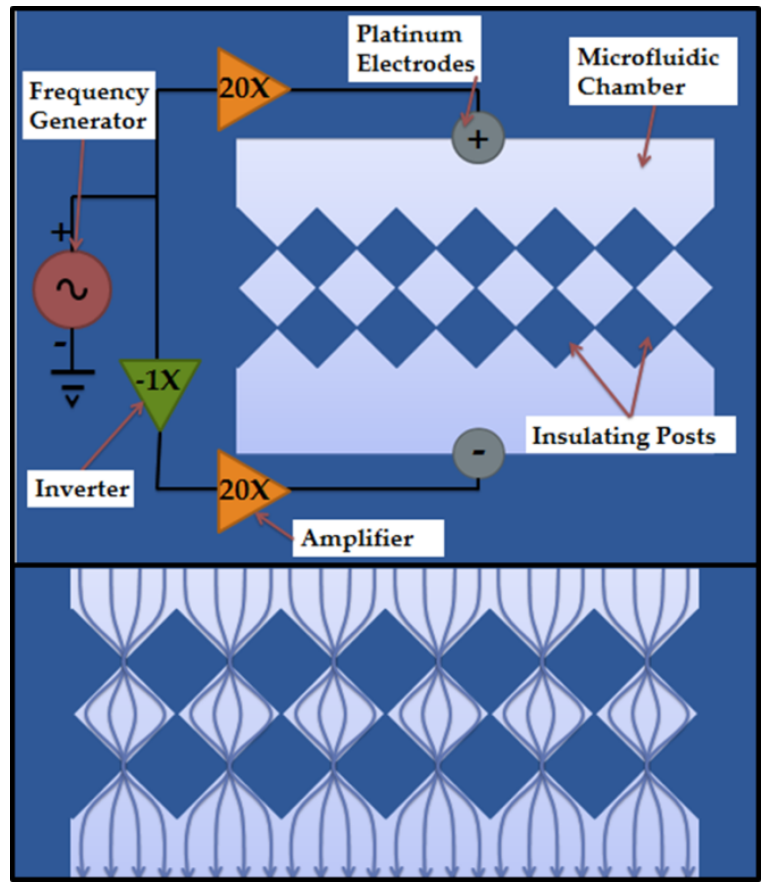

Figure 15: A: (top) electrical connections for constriction DEP devices. B:(bottom) electric field lines in the Diamond post design

silicon master. Following a vacuum degassing step, the mold was allowed to harden overnight on a hot plate. The cured PDMS was then carefully released from the Silicon master leaving the microfluidic design molded on its surface. The PDMS mold was then diced into single chips, and inlet and outlet holes were drilled with a punch. After a cleaning step to remove drilling debris, a 
low energy oxygen plasma environment was used to bond the PDMS channels to a glass microscope coverslip. DI water containing the oocyst sample was then loaded through the drilled inlet and outlet channels, and the platinum electrodes were inserted. As is shown in figure 15A a 40x amplifier was used in series with the waveform function generator, to obtain the high voltage necessary for EDEP,. Due to the high impedance of the mircrofluidic chip, it was necessary to calibrate the signal generator for a High-Z load.

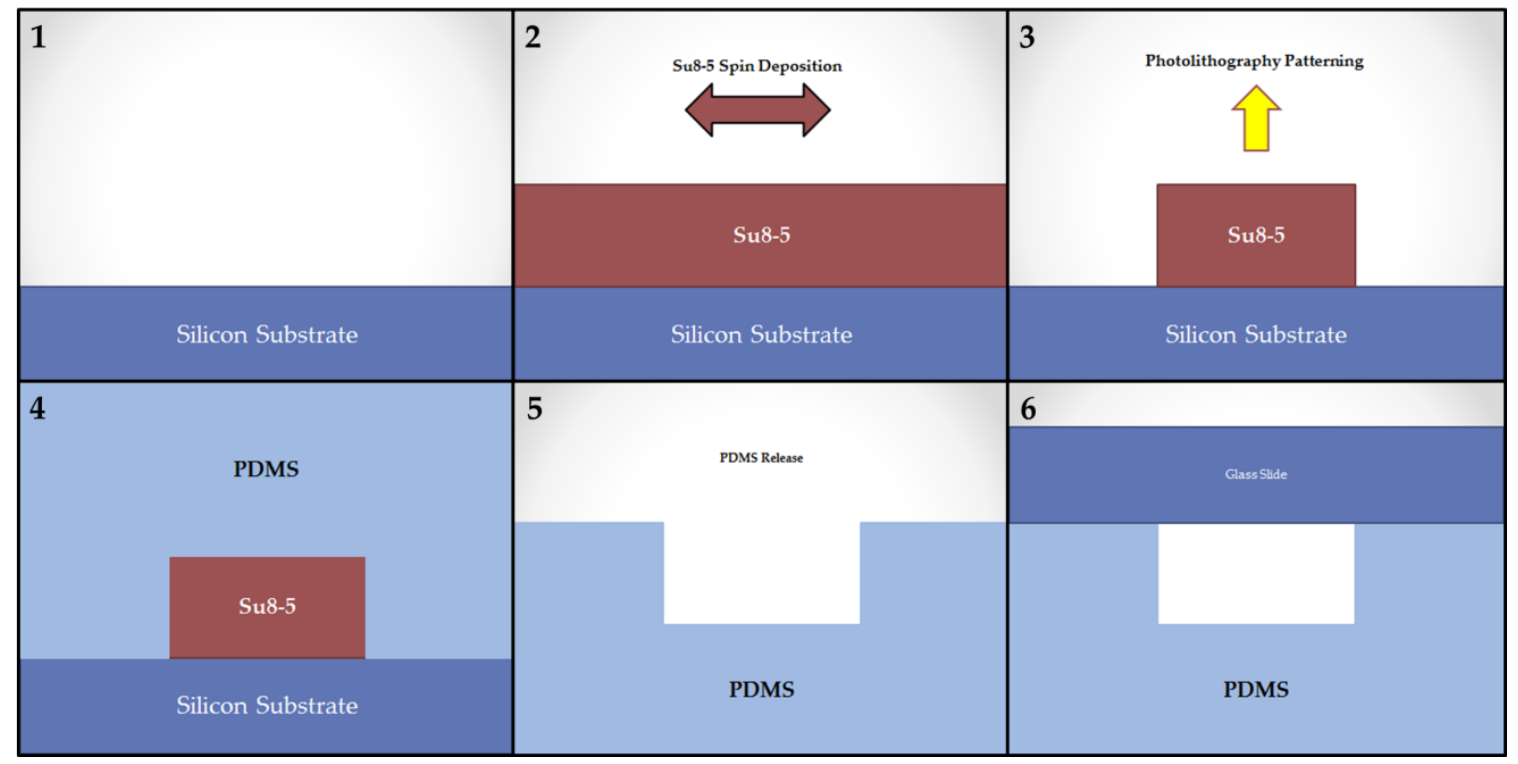

Figure 16: process flow for Constriction Chip Fabrication

\section{Results: Diamond Post Design}

Various versions of these diamond post designs were fabricated with different post sizes, constriction slopes, and numbers of rows and columns in an attempt to find an optimal setup. Unfortunately most of the fabricated designs failed to produce any substantial DEP response due to flaws in the design and fabrication method. The failure of many of these chips can be traced to the use of printed Mylar masks during the photolithographic patterning of the Su8-5 masters. These masks, used due to their low cost and fabrication speed, tended to result in rough or jagged constriction walls creating parasitic high field points. Furthermore, they would often result in 
inconsistent constriction channel widths ranging from $5 \mathrm{um}$ to $40 \mathrm{um}$. Also they often resulted in rounded constriction tips, which minimized the magnitude of field in the constriction, most likely causing weaker DEP trapping. However, two designs were able to produce both strong positive and negative DEP responses. The first was a seven columns by two row device with approximately 15 um constrictions with a $90^{\circ}$ slope. The second was three row by four columns device with $38 \mathrm{um}$ constrictions with a $30^{\circ}$ slope.

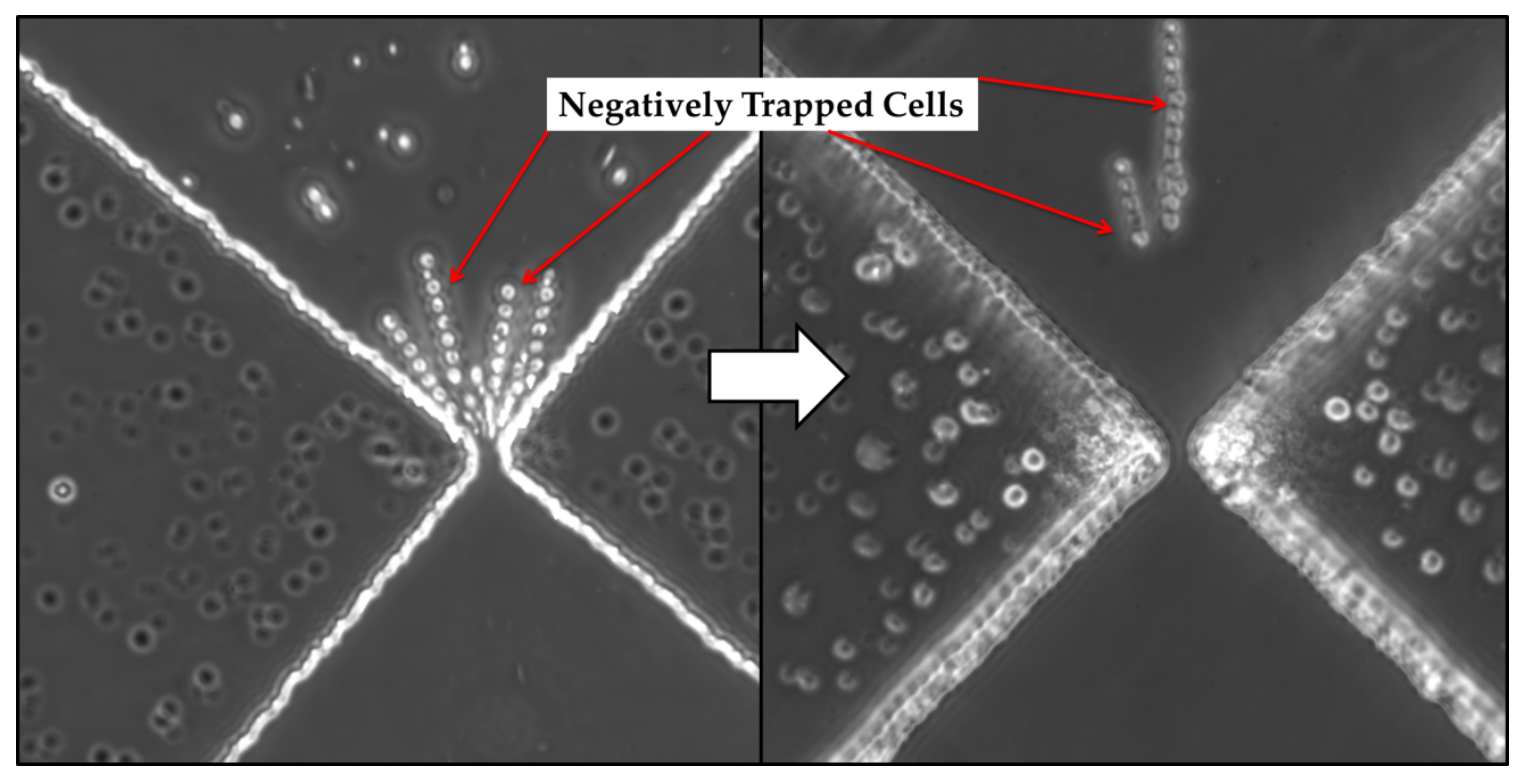

Figure 17: Strong Negative trapping in the 7X2 diamond post device.

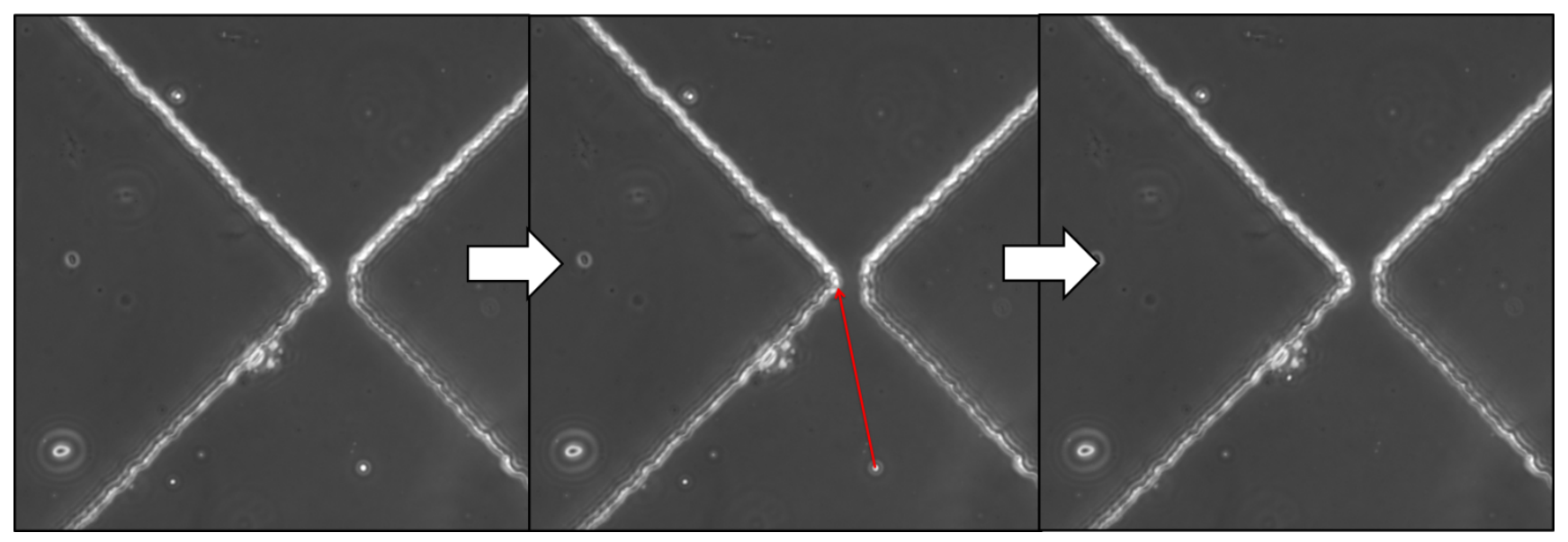

Figure 18: Positive trapping in the 7X2 diamond post device. The left image shows the initial position of the Cryptosporidium Oocysts. The red lines in the center image indicate the direction of translation. The right image shows the final position of the Cryptosporidium Oocysts 


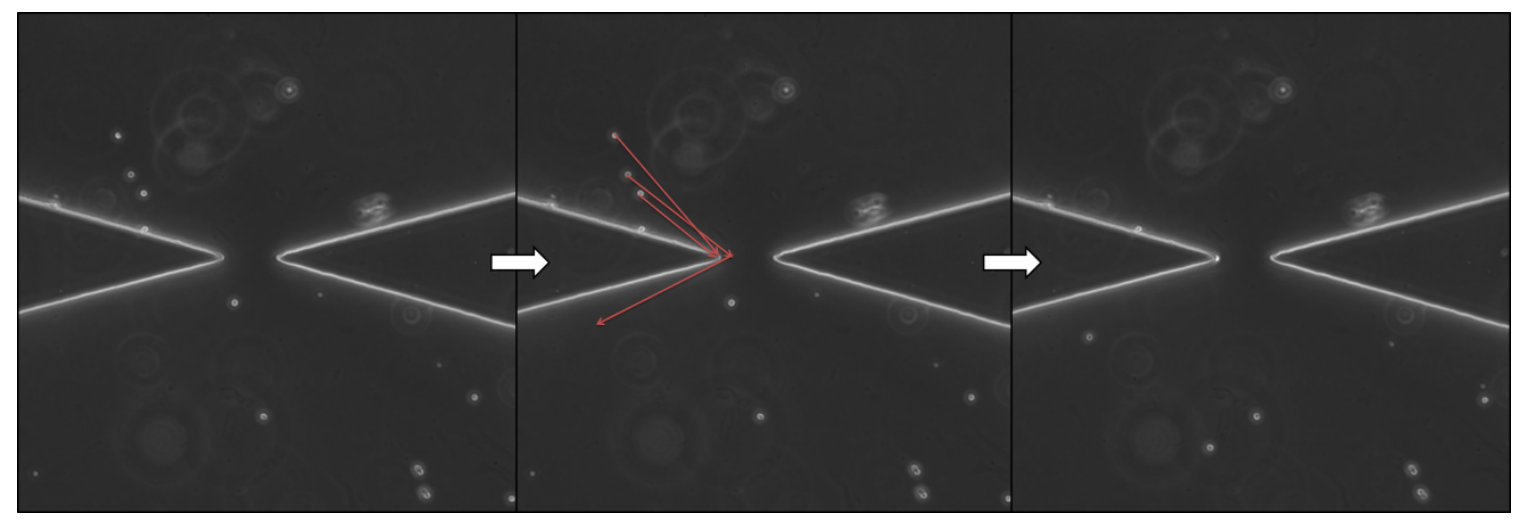

Figure 19: Positive trapping in the $3 \times 4$ device. The left image shows the initial position of the Cryptosporidium Oocysts. The red lines in the center image indicate the direction of translation. The right image shows the final position of the Cryptosporidium Oocysts.

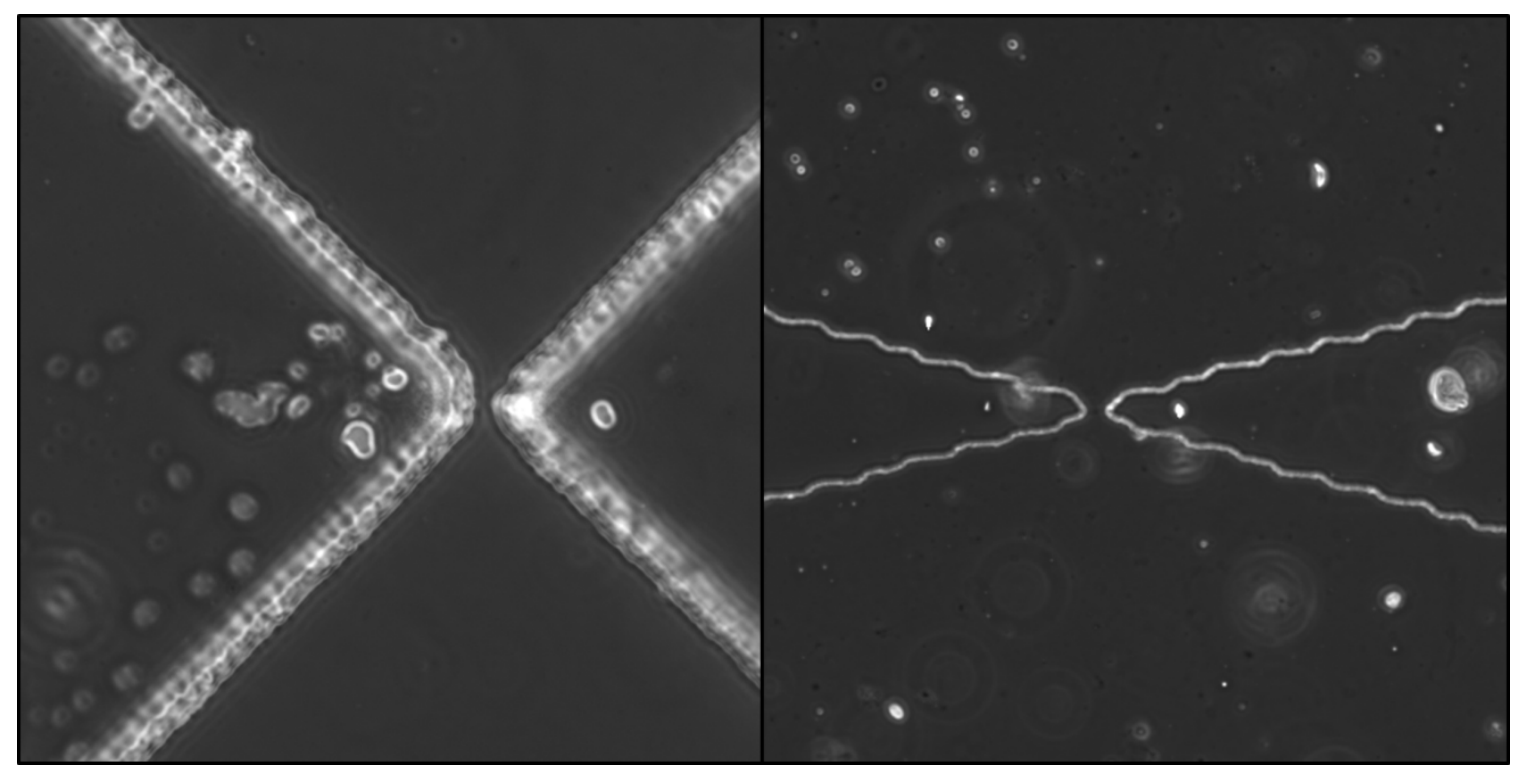

Figure 20: Image detailing some of the edge defects caused by the use of Mylar printed masks. Note the rounded and rough edges in the image to the left and the jagged edges in the image to the right.

\section{Design: Single channel Constriction EDEP Device}

The second EDEP device utilized a single microfluidic channel containing nine constrictions in series. While a single channel device design lowers sample throughput in comparison to the multiple channels of the diamond post design, it would be able to obtain a similar profile of the applied field -for a lower current drawn from the electrical signal generator. This can be explained by comparing the circuit model of a multi-channel device to that of a single channel device. While the single channel device offers only a single path for the input 
current to follow, a multi-channel device can be modeled as a parallel circuit, dividing the input current evenly between each of the

channels. Hence, for the case of a

multichannel device, a much higher input

current will be needed to apply the same

electric field.

For this design the channel width is $1.5 \mathrm{~mm}$,

which constricts to a $10 \mu \mathrm{m}$ gap over an

extent of $0.25 \mathrm{~mm}$. Again a standard

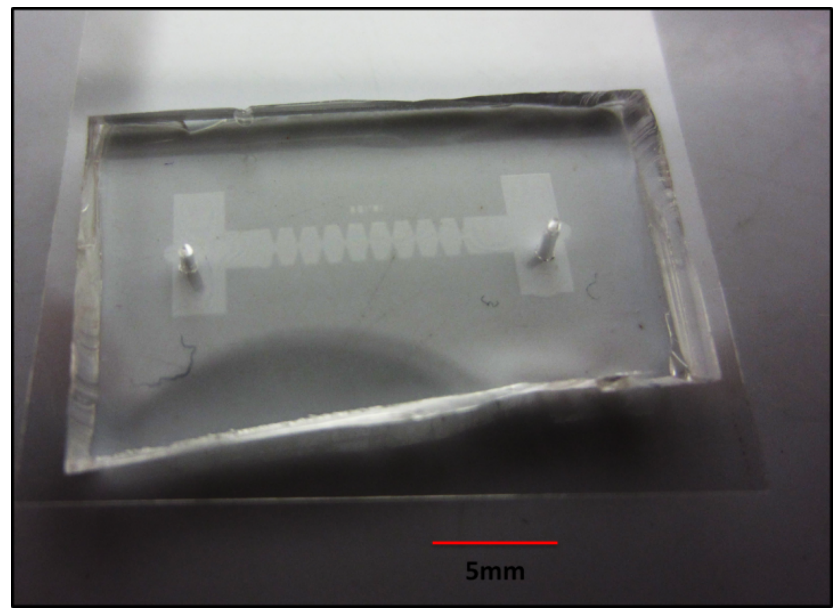

Figure 10: image of the single channel device. Contrast raised to $55 \%$ to increase visability

channel depth of $8 \mu \mathrm{m}$ was used. The gradient was designed to be steeper than was initially used in the prior design of the diamond post. In this manner, the DEP trapping force can be enhanced through enhancing field gradients, since the constriction ratio was limited to 150 -fold (1500 $\mu \mathrm{m}$ to $10 \mu \mathrm{m})$, by the need to have constriction gaps of $\sim 10 \mu \mathrm{m}$ or larger. To account for the smaller region of high-field non-uniformity due to the lowered spatial extent of the constriction, electrophoresis is utilized to transport the oocysts to the proximity of the constriction. To facilitate this electrophoreticaly enhanced DEP action, larger reservoirs have been included at the inlet and outlet. The channel length separating between the constrictions was designed to be $0.5 \mathrm{~mm}$ to provide a larger sample volume for each constriction, for utilizing DC electrophoresis transport of the oocysts towards the constriction. To enhance the accuracy of the microfabricated features, the glass mask was constructed using chromium on soda lime for generating the Su8-5 on silicon master. The improved quality of the patterning step resulted in sharper and more consistent constriction features. Also it resulted in far more uniform channel walls. 


\section{Results: Single Channel Device}

During experimentation with untreated Cryptosporidia oocysts, strong negative DEP was observed from $1 \mathrm{KHz}$ to $200 \mathrm{kHz}$ and strong positive DEP was observed from $350 \mathrm{kHz}$ to $1 \mathrm{MHz}$. At higher frequencies, the DEP force was reduced to a level below the noise floor. This suggests the first crossover frequency (negative to positive DEP) of between $200 \mathrm{kHz}$ and $350 \mathrm{kHz}$; and a possible secondary crossover frequency (positive to negative) somewhere above $1 \mathrm{MHz}$. With these devices two categories of heat treated oocysts were tested. The first category was treated at $80^{\circ} \mathrm{C}$ for 5 minutes. These oocysts showed a relatively weak level of negative DEP trapping at frequencies below $200 \mathrm{kHz}$. Above $200 \mathrm{kHz}$ the negative DEP appeared to grow weaker until tapering off completely in the MHz range. The second category of heat treated oocysts was heated to the higher temperature of $100{ }^{\circ} \mathrm{C}$ for 5 minutes. These samples demonstrated strong positive DEP from $1 \mathrm{KHz}$ onwards, which appears to gradually fall-off from $400 \mathrm{kHz}$ onwards to a level of no discernable DEP behavior at $1 \mathrm{MHz}$. Based on the DEP spectra of untreated oocysts, the inverse RC time constant of an intact oocyst wall is at $\sim 400 \mathrm{kHz}$. Hence, the difference between the frequency response of the two heat treated specimens can be attributed to the ionic permeability of their oocyst wall for DEP behavior at frequencies below $400 \mathrm{kHz}$ and to the cytoplasm conductivity for DEP behavior at frequencies above $400 \mathrm{kHz}$. In the case of the $80^{\circ} \mathrm{C}$ sample, the oocyst wall, while damaged, was likely able to provide some level of capacitive shielding, thereby causing negative DEP at frequencies less than $400 \mathrm{kHz}$. In the case of the heat treated sample at $100^{\circ} \mathrm{C}$, the oocyst wall was likely to have been completely destroyed, which prevented any substantial capacitive shielding. Hence, the positive DEP behavior at low frequencies, which falls off beyond $100 \mathrm{kHz}$, may be attributed to the high surface conductance of ions through a permeable oocyst wall that is destroyed due to heat treatment. 


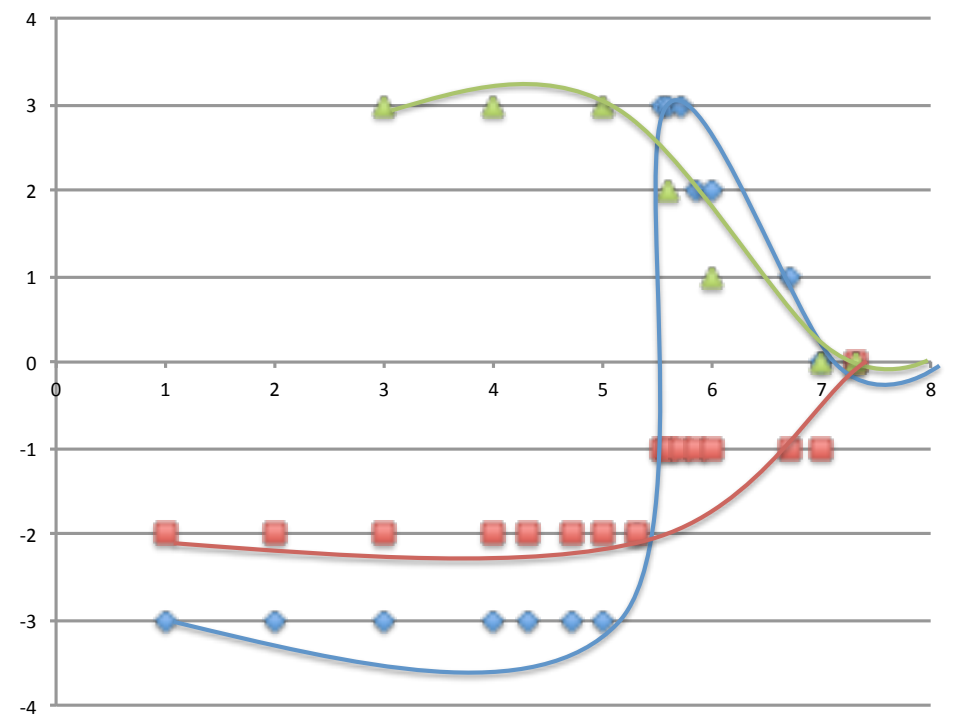

- Untreated - DI(constriction)

$\square$ Heat Treatment (80 C) -DI (constriction)

$\triangle$ Heat Treatment (100C) - Di

Figure 22: qualitative DEP strength and direction version the log scale of the applied AC electric field for untreated and Heat treated cryptosporidium oocytes in a single channel constriction device.

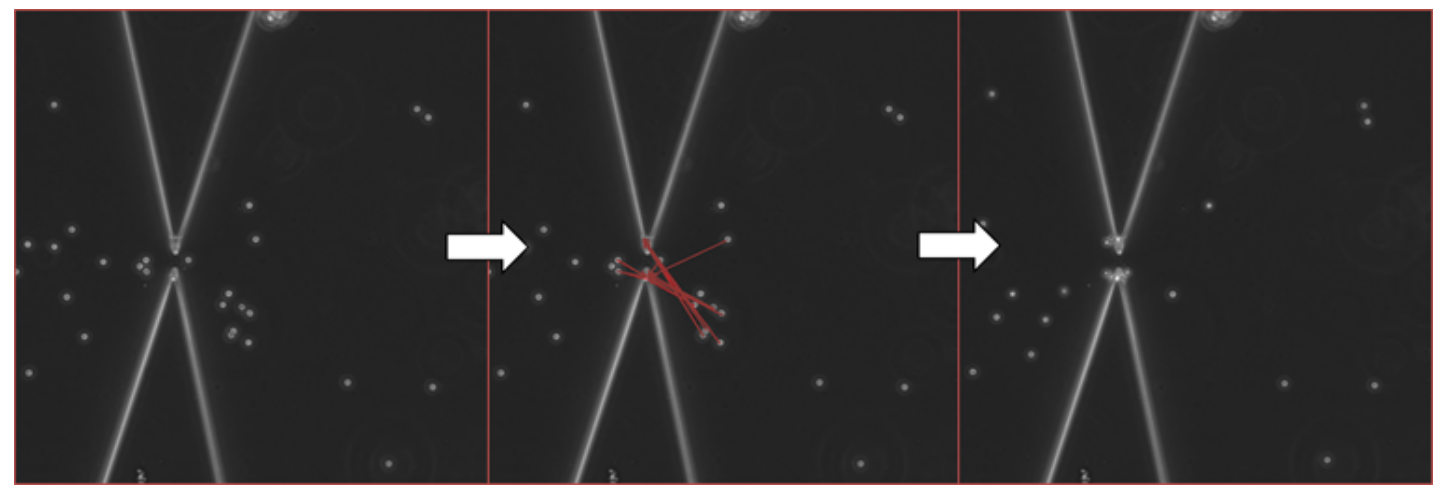

Figure 23: An example of strong positive trapping in the single channel constriction device. The left image shows the initial position of the Cryptosporidium Oocysts. The red lines in the center image indicate the direction of translation. The right image shows the final position of the Cryptosporidium Oocysts

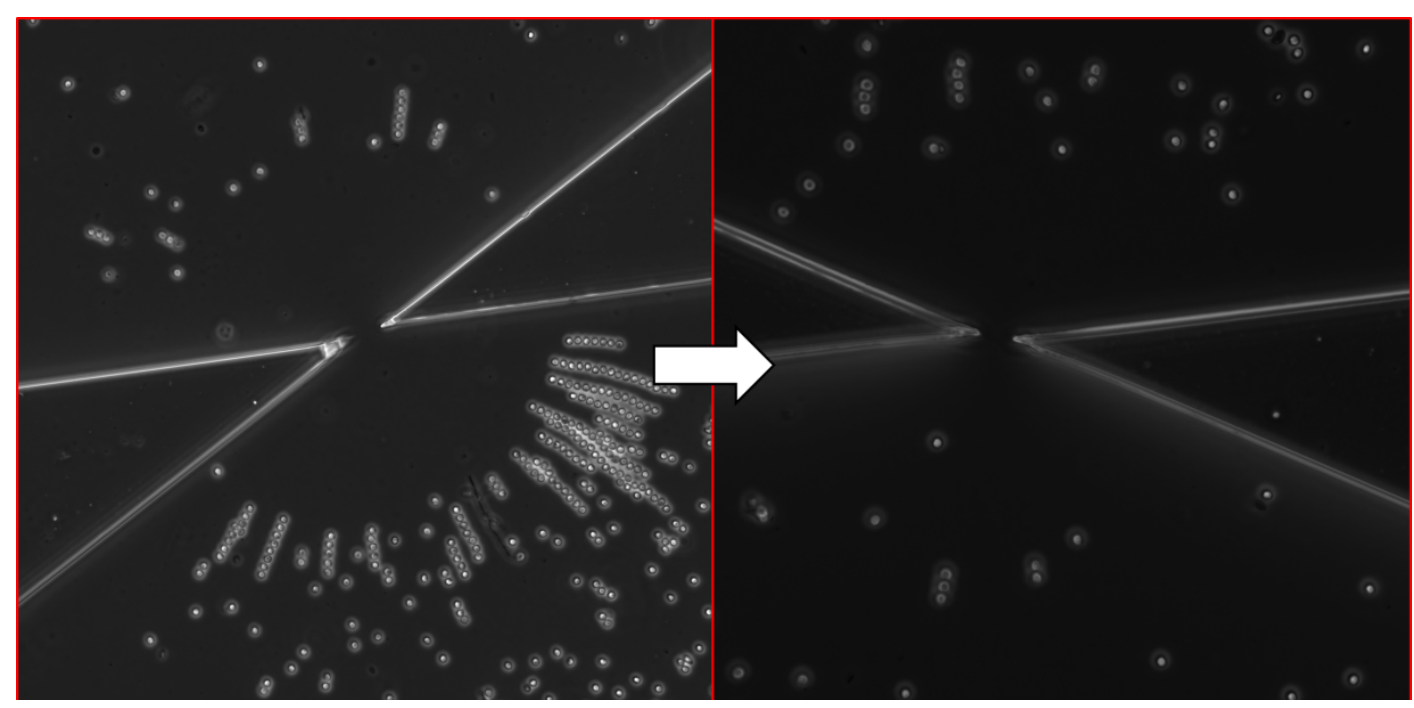


While DI water media was used in the prior experiments, it is necessary to be able to conduct the dielectrophoresis experiments within media of higher ionic strength to simulate native water environments and to reduce the osmotic pressure on biological cells. We would anticipate that the increase in medium conductivity $\left(\sigma_{\mathrm{m}}\right)$ upon the addition of salt is likely to reduce the magnitude of positive DEP force due to lower CM factor (i.e. lower: $\sigma_{\mathrm{p}}-\sigma_{\mathrm{m}}$ ), as well as to shift the inverse $\mathrm{RC}$ time constant to higher frequencies. This reduction in magnitude of positive DEP and shifting of the frequency response to higher frequencies is within figure 7, showing experimental data on the DEP frequency response at differing $\mathrm{NaCl}$ levels. In the case of the lower crossover frequency, which is often attributed to the frequency at which the capacitive oocyst wall no longer shields the higher conductivity cytoplasm, this shift may be explained by a change in the conductance of the medium relative to the conductance of the particle. As equation 2 for the $\mathrm{CM}$ factor demonstrates, for positive DEP to occur the effective permittivity of the medium must be lower than the effective permittivity of the particle. As equation 3 demonstrates, at low frequencies the effective permittivity of medium and the particle are largely governed by their conductance. For positive DEP to occur at relatively low frequencies, the conductivity of the particle must be higher than that of the medium. As has been previously discussed, at very low frequencies the capacitive action of the oocyst wall for a viable cryptosporidium oocyst will shield the high conductivity cytoplasm, thereby delaying positive DEP to later frequencies. As the frequency is increased, the shielding effect of the cell wall decreases, thereby increasing the overall conductivity of the particle, which is now dominated by that of the high-conductivity cytoplasm region. The RC time constant of the oocyst wall is given by: $\tau \sim\left(\mathrm{C} / \sigma_{\mathrm{p}}\right)($ length/area $)$. For DEP to occur at low medium conductivity $\left(\sigma_{\mathrm{m} 1}\right)$, the minimum necessary effective particle conductivity $\left(\sigma_{\mathrm{p} 1}\right)$ is lower than the particle conductivity $\left(\sigma_{\mathrm{p} 2}\right)$ 
required to cause positive DEP with a higher conductivity media $\left(\sigma_{\mathrm{m} 2}\right)$ : i.e. since $\sigma_{\mathrm{m} 2}>\sigma_{\mathrm{m} 1}$; hence for observing positive DEP, the minimum required $\sigma_{\mathrm{p} 2}$ exceeds $\sigma_{\mathrm{p} 1}$. Hence, the respective RC time constant observed for the oocyst wall for DEP behavior in high conductivity medium $\left(\tau_{2}\right)$ is smaller than that within media of lower conductivity $\left(\tau_{1}\right)$, thereby causing the crossover from negative to positive DEP to occur at higher frequencies with increasing salt levels (i.e. $\omega_{2}>$ $\left.\omega_{1}\right)$. It is also noteworthy that by observing DEP behavior within media of low and high conductivity using the constriction EDEP device, we have demonstrated its versatility for application within media of a wider range of conductivities. This is important, since the positive DEP action of moderately conducting biological particles is usually harder to observe within media of high conductivity due to the lower ensuing DEP forces, as a result of the smaller CM factor and the higher capacitive screening of the applied fields at low frequencies due to the double-layer around the electrodes. In the constriction EDEP device, we have avoided the latter

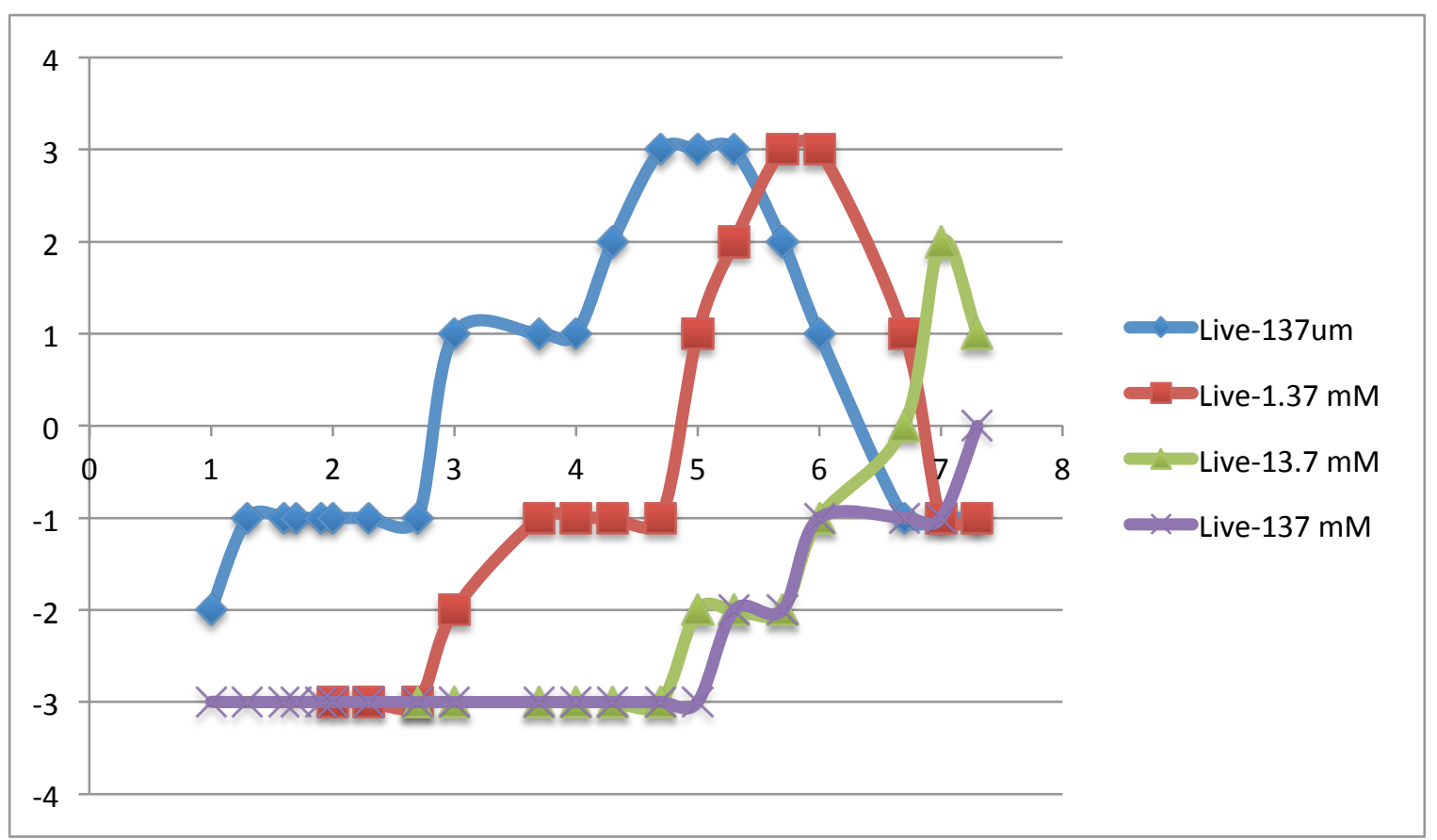

Figure 25: : qualitative DEP strength and direction version the log scale of the applied AC electric field for untreated and Heat treated cryptosporidium oocytes in a single channel constriction device for various levels of salt concentration. 
problem by creating high-field points that are not localized on insulating constriction tips, rather than at the electrodes tips, where the high capacitance double-layer can screen this field. The former problem is circumvented by enhancing the constriction ratio and sharpness to enhance DEP force. On the other hand, using the quadrapole DEP device within media of highconductivity, screening of field and electrolysis can prevent the observation of DEP behavior at low frequencies up to $\sim 500 \mathrm{kHz}$.

\section{Conclusion}

A comparison of the DEP frequency responses of untreated versus disinfectant treated Cryptosporidia oocysts using the quadarapole device and the single channel constriction EDEP device demonstrates the strengths of each design for the characterization of DEP behavior. While the quadapole was largely unable to gather discernable data in the low frequency range (1kHz$200 \mathrm{kHz}$ ) the single channel constriction device was able to consistently do so. For cell viability testing, accuracy in this range is vital, since the DEP response at these frequencies will help characterize the ion permeability cell membrane or oocyst wall based on its inverse RC time constant. The results from the single channel constriction EDEP device demonstrate an ability to make discernable distinctions between untreated, low temperature heat treated $(80 \mathrm{C})$ and boiling temperature heat treated $(100 \mathrm{C})$ oocysts in low frequency range $(1 \mathrm{kHz}-200 \mathrm{kHz})$.

At the mid frequency range $(200 \mathrm{kHz}-1 \mathrm{MHz})$ both these devices operated relatively well for DEP characterization within media of low salt, as apparent from their similar crossover frequencies from negative to positive DEP. With its higher field strength, due to the close proximity of its energizing electrodes, the quadrapole device did seem better suited to generating a negative DEP response in this frequency range. For purposes of quantifying the DEP response, the EDEP constriction device proved more flexible, allowing for more efficient transportation of 
oocyst towards the area of interest at the constriction, through utilizing DC electrophoresis. On the other hand, for the quadrapole device, the opaque nature of the gold on titanium microelectrodes and the tendency of the oocysts to irreversibly absorb onto the electrodes during positive DEP trapping limited the acquisition of quantitative DEP data after the respective disinfection treatments.

Finally at the high frequency range $(1-10 \mathrm{MHz})$ both these devices demonstrated significantly different DEP responses. While the oocysts seemed to exhibit positive DEP action at these frequencies on quadrapole devices, the DEP action was diminished on the constriction EDEP devices, with no discernable DEP response beyond $5 \mathrm{MHz}$. The reason for this disparity could be attributed to two separate causes. In order to generate the high voltage necessary for EDEP the constriction device needs to use a $40 \mathrm{X}$ amplifier to boost the output signal of the frequency generator. While efficient at amplifying frequencies below $1 \mathrm{MHz}$, the gain of this amplifier diminishes for higher frequency input signals. This means less voltage will be supplied to the device at these frequencies. If this gain drops low enough then it will prevent any DEP response at frequencies greater than $1 \mathrm{MHz}$, and this may explain the drop-off in positive DEP of the oocysts on the EDEP device beyond $1 \mathrm{MHz}$. On the other hand, the signal energizing the quadrapole device has no need for amplification, due to the close proximity of the electrodes and hence, positive DEP behavior of the oocysts is apparent on the quadrapole device, even at frequencies up to $5 \mathrm{MHz}$. Another possible cause for the difference between the high frequency response of these two devices is the poor dynamic range of the quadrapole device. In this context, dynamic range refers to the ability to easily determine differences between weak and strong DEP response. The high magnitude of the high field points of the quadrapole device, cause a similar level of DEP translation under strong and weak DEP polarization. This may lead 
to the false conclusion that the DEP force has remained unchanged while it has in fact

diminished in a similar manner as the constriction device. It is also noteworthy that the DEP behavior of the oocysts could be quantified in media of a wide range of conductivities using the constriction EDEP device, whereas the DEP behavior could only be ascertained in DI water using the quadrapole DEP device, since field screening at low frequencies $(<500 \mathrm{kHz})$ reduces the trapping level of the oocysts.

\section{Works Cited:}

[1] C. Zhang, K. Khoshmanesh, A. Mitchell, and K. Kalantar-zadeh, "Dielectrophoresis for manipulation of micro/nano particles in microfluidic systems," Analytical and Bioanalytical Chemistry, vol. 396, pp. 401-420, Jan 2010.

[2] H. Bridle, M. Kersaudy-Kerhoas, B. Miller, D. Gavriilidou, F. Katzer, E. A. Innes, et al., "Detection of Cryptosporidium in miniaturised fluidic devices," Water Research, vol. 46, pp. 1641-1661, Apr 152012.

[3] B. J. King and P. T. Monis, "Critical processes affecting Cryptosporidium oocyst survival in the environment," Parasitology, vol. 134, pp. 309-323, Mar 2007.

[4] H. Morgan and N. G. Green, AC electrokinetics : colloids and nanoparticles. Baldock, Hertfordshire, England

Philadelphia, Pa.

Williston, VT: Research Studies Press ;

Institute of Physics Pub. ;

Distribution, North America, AIDC, 2003.

[5] J. Regtmeier, R. Eichhorn, M. Viefhues, L. Bogunovic, and D. Anselmetti, "Electrodeless dielectrophoresis for bioanalysis: Theory, devices and applications (vol 32, pg 2253, 2011)," Electrophoresis, vol. 32, pp. 3090-3090, Nov 2011.

[6] A. D. Goater and R. Pethig, "Electrorotation and dielectrophoresis," Parasitology, vol. 117, pp. S177-S189, 1998.

[7] R. Fayer, "Effect of High-Temperature on Infectivity of Cryptosporidium-Parvum Oocysts in Water," Applied and Environmental Microbiology, vol. 60, pp. 2732-2735, Aug 1994.

[8] R. Pethig and G. H. Markx, "Applications of dielectrophoresis in biotechnology," Trends in Biotechnology, vol. 15, pp. 426-432, Oct 1997.

[9] R. Pethig, "Dielectrophoresis: Status of the theory, technology, and applications (vol 4, 022811, 2010)," Biomicrofluidics, vol. 4, Sep 2010.

[10] P. R. C. Gascoyne and J. Vykoukal, "Particle separation by dielectrophoresis," Electrophoresis, vol. 23, pp. 1973-1983, Jul 2002. 
[11] Z. R. Gagnon, "Cellular dielectrophoresis: Applications to the characterization, manipulation, separation and patterning of cells," Electrophoresis, vol. 32, pp. 24662487, Sep 2011.

[12] P. Patel and G. H. Markx, "Dielectric measurement of cell death," Enzyme and Microbial Technology, vol. 43, pp. 463-470, Dec 102008.

[13] C. M. Quinn, G. P. Archer, W. B. Betts, and J. G. ONeill, "Dose-dependent dielectrophoretic response of Cryptosporidium oocysts treated with ozone," Letters in Applied Microbiology, vol. 22, pp. 224-228, Mar 1996.

[14] H. N. Unni, D. Hartono, L. Y. L. Yung, M. M. L. Ng, H. P. Lee, B. C. Khoo, et al., "Characterization and separation of Cryptosporidium and Giardia cells using on-chip dielectrophoresis," Biomicrofluidics, vol. 6, Mar 2012. 